\title{
Mining subgraph coverage patterns from graph transactions
}

\author{
A. Srinivas Reddy ${ }^{1}$ (D) P. Krishna Reddy ${ }^{1} \cdot$ Anirban $^{\text {Mondal }}{ }^{2} \cdot$ U. Deva Priyakumar $^{1}$
}

Received: 23 February 2021 / Accepted: 24 October 2021 / Published online: 2 December 2021

(c) The Author(s), under exclusive licence to Springer Nature Switzerland AG 2021

\begin{abstract}
Pattern mining from graph transactional data (GTD) is an active area of research with applications in the domains of bioinformatics, chemical informatics and social networks. Existing works address the problem of mining frequent subgraphs from GTD. However, the knowledge concerning the coverage aspect of a set of subgraphs is also valuable for improving the performance of several applications. In this regard, we introduce the notion of subgraph coverage patterns ( $S C P s)$. Given a GTD, a subgraph coverage pattern is a set of subgraphs subject to relative frequency, coverage and overlap constraints provided by the user. We propose the Subgraph ID-based Flat Transactional (SIFT) framework for the efficient extraction of SCPS from a given GTD. Our performance evaluation using three real datasets demonstrates that our proposed SIFT framework is indeed capable of efficiently extracting SCPs from GTD. Furthermore, we demonstrate the effectiveness of SIFT through a case study in computer-aided drug design.
\end{abstract}

Keywords Graph mining $\cdot$ Subgraph mining $\cdot$ Subgraph coverage patterns $\cdot$ Bio-informatics

\section{Introduction}

A complex graph can be built from pieces of knowledge based on the relationships among various entities. Such a graph contains new kinds of interesting and useful knowledge structures. Hence, it can be extremely valuable for opening up new avenues for enhancing applications in several domains. In this regard, graph mining $[10,33]$ has become an active area of research for mining knowledge from graph representations in bio-informatics, chemical informatics, social networks, computer vision, video indexing, text retrieval and web analysis. Mining the knowledge of frequent subgraphs from graph transactional data (GTD) is an important and active area of graph mining [14,17,22,23,40,42]. It has been demonstrated in $[29,34]$ that frequent subgraph mining can

A. Srinivas Reddy

srinivas.annappalli@research.iiit.ac.in

P. Krishna Reddy

pkreddy@iiit.ac.in

Anirban Mondal

anirban.mondal@ashoka.edu.in

U. Deva Priyakumar

deva@iiit.ac.in

1 Kohli Centre on Intelligent Systems, IIIT, Hyderabad, India

2 Department of Computer Science, Ashoka University, Delhi, India extract interesting patterns from GTD for providing valuable knowledge in the domain of bio-informatics.

Knowledge concerning the coverage aspect of a set of subgraphs can be valuable for improving the performance of several applications. In the literature, the notion of coverage has been well explored in set theory and graph theory [7, $11-13,15,19,27,39,47]$ as well as the extraction of coverage patterns from transactional data $[18,36]$. However, none of the existing works have investigated the issue of extracting the coverage-related knowledge of patterns from GTD. We believe that the coverage-related knowledge in the form of subgraph patterns can be used in improving the performance of applications in chemical, biological and social network domains.

Computational biology approaches have become ubiquitous in the process of discovering new drug molecules that can treat diseases. In the process of drug design, careful and systematic changes in the molecular structure, which can maximize the interactions between the drug molecule and the protein relevant to the given disease, are crucial. Methods that help us to understand and quantify such intermolecular interactions between proteins and drug molecules will open up significant avenues for research in this direction. In the literature, frequent subgraph mining (FSM) techniques have been applied in $[29,34]$ to study protein-ligand interac- 
tions by analyzing the interaction patterns of different drug molecules with the target protein.

Consider a scenario, where we have identified a number of low-binding drug molecules. The objective is to improve the structure of the molecules to increase the binding affinity so that the drug would work at lower dosages. Existing FSM techniques are not capable of extracting coverage-related knowledge of patterns. However, if we develop approaches for discovering coverage-related knowledge patterns, such methods can be effectively used to help in optimizing the binding affinity of the drug molecules w.r.t. the selected protein, thereby making the drug design process more efficient.

A subgraph coverage pattern $(S C P)$ is a set (or pattern), whose elements are the subgraphs of GTD. This work addresses the problem of extracting all SCPS, which cover a given percentage of the graph transactions of GTD. Notably, in addition to the coverage aspect, we have to consider the aspect of overlap, which arises as a consequence of considering the coverage of a set of subgraphs. The sets of graph transactions covered by the corresponding subgraphs of an $S C P$ may contain the common transactions, which we refer to as overlap. In addition to coverage, we consider an SCP is interesting if there is a minimal overlap among the graph transactions covered by subgraphs of an $S C P$.

Given a GTD, the issue is to extract all of the possible $S C P$ s subject to the constraints associated with coverage and overlap. A brute-force approach would be to first extract all of the possible subgraphs of GTD by employing a frequent subgraph extraction algorithm $[9,22,25,42]$ and then determine the coverage and overlap for each possible pattern consisting of subgraphs. Intuitively, such an approach would be prohibitively expensive because the number of possible subgraphs in a given GTD typically explodes. Consequently, the number of candidate patterns to be considered for the extraction of $S C P S$ also explodes.

For efficiently extracting SCPs from a given GTD, we introduce the model of $S C P s$ and present the framework to extract SCPS. In the proposed model, we define the notion of coverage and overlap and present the problem to extract $S C P$ s. The problem is to extract all SCPs from a given GTD by satisfying the threshold values of given coverage and overlap. We present a framework to extract $S C P$, which we designate as subgraph-identifier-based flat transactional (SIFT) framework. As a part of SIFT framework, we propose an approach to extract all subgraphs of the graph transactions in GTD and assign a unique subgraph identifier (SID) to each subgraph. Next, each graph transaction in GTD is transformed into the corresponding flat transaction, which consists of the corresponding SIDs. We also propose an approach to extract SCPS from the flat transactional dataset. The problem is similar to that of coverage pattern extraction [36] from flat transactional databases subject to coverage and overlap constraints. Incidentally, overlap follows the sorted closure property [28], which we shall use in this work for facilitating effective pruning. Hence, we extend the existing pattern extraction approach [36], which employs pruning strategy based on overlap, for the efficient extraction of SCPs. Observe that by forming a set of flat transactions, complex computationally intensive graph operations associated by coverage and overlap are replaced with the corresponding simpler and relatively fast set-based operations.

The key contributions of this work are three-fold:

1. We introduce the notion of subgraph coverage patterns $(S C P s)$ for GTD.

2. We propose the SIFT framework for efficiently extracting $S C P$ from a given GTD.

3. We conduct an extensive performance study using three real datasets to demonstrate that it is indeed feasible to efficiently extract SCPS using our proposed SIFT framework. We also demonstrate the effectiveness of SIFT through a case study in computer-aided drug design.

To the best of our knowledge, this is the first work to consider the extraction of subgraph coverage patterns from graph transactional data. The remainder of this paper is organized as follows. Section 2 reviews related works and background information. Section 3 discusses the proposed framework of the problem. Section 4 presents our proposed SIFT framework. Section 5 reports our performance evaluation. Section 6 presents our case study. Finally, we conclude in Sect. 7.

\section{Related work and background}

This section discusses related works and background.

\subsection{Related work}

Research efforts, such as the gindex approach [44], have designed indexes for extracting subgraphs by considering line, cycle and star as basic graph query structures. Moreover, the GString semantic-based approach [24] indexes chemical compounds databases.

Graph mining techniques have also been applied in GTDs. The work in [22] used an apriori-based algorithm for discovering frequent subgraphs in GTDs. Moreover, the work in [25] discussed the frequent subgraph mining algorithm, which incorporates canonical labelling in conjunction with sparse graph representation to reduce both time and space complexity. The work in [42] proposed the gSpan algorithm for discovering frequent subgraphs without candidate generation. In particular, gSpan uses a lexicographic ordering for mapping each graph to a unique minimum depth-first-search code as its canonical label. A good survey on frequent sub- 
graph mining techniques for GTDs can be found in [23]. An algorithm to extract the top- $k$ frequent subgraphs has been proposed in [17].

The work in [26] proposed REAFUM, an approximate subgraph mining framework that constructs a list of representative graphs. It extracts frequent representative subgraphs, allows approximate matches and extracts consensus patterns. Another work in [48] proposed HOS-PLOC, a local clustering framework that extracts a small high-order conductance cluster, which largely preserves the user-specified network structures.

The work in [9] proposed an algorithm to mine molecular fragments based on association rule mining. These molecular fragments help in discriminating drug classes. Researchers have made efforts to model protein-ligand complex (PLC) as graph structures and extracted frequently occurring subgraph patterns. The works in [34,35] proposed GReMLIN (graph mining strategy to infer protein-ligand interaction patterns), which is a methodology to search for conserved protein-ligand interactions in a group of related proteinsligand complexes. They use frequent subgraph mining to recognize structural patterns relevant to protein-ligand interaction. The work in [29] modeled PLC as a bipartite graph and used graph topological properties like degree, closeness, communicability, eccentricity, node betweenness and edge betweenness to summarize and extract frequent patterns. The work in [40] proposed FERRARI, which is a visual exploratory subgraph search framework. In particular, it employs two index structures VACCINE and ADVISE for indexing frequent and infrequent subgraphs to improve efficiency and scalability.

The notion of coverage has been well explored in set theory in the form of the set cover problem [12] and the hitting set problem [15]. In graph theory, the notion of coverage has been explored in the form of the minimum vertex cover problem [11,39], hitting set problem in hypergraph, traversals of a hypergraph [20], clique cover problem [19] and influence maximization problem [27,47]. The notion of coverage has been used in hypergraphs in the form of the minimum hitting set problem [7], which involves the extraction of the set of vertices that have a non-empty intersection with every hyperedge. The work in [31] states that a set of $k$-mers is a universal hitting set if every possible $L$-long sequence contains a $k$-mer from a given DNA/RNA sequence dataset.

Clique cover is another important problem with applications in compiler optimization, computational geometry and applied statistics [19]. Information coverage maximization in social networks $[27,47]$ also uses coverage. An approach was proposed in [46] to select a set of influential users. More recently, the work in [41] proposed TOPKLS, a local search algorithm for finding diversified top- $k$ cliques from a given graph. The notion of coverage has been well explored to solve the maximum coverage problem in facility location
[6]. However, these works explore the notion of coverage for a single graph as opposed to a GTD, which is our focus. Moreover, the works in $[18,36]$ find coverage patterns in transactional data using pattern-growth and level-wise pruning approaches, respectively.

Notably, all of the existing works have addressed the issue of GTDs for graph search and mining of frequent subgraphs related knowledge with applications in chemical and biological areas. The issue of extracting the knowledge related to coverage from GTD has not been addressed. In contrast with the existing works [29,40,42], in this paper, we have made an effort to propose an approach to extract coverage-related knowledge from GTDs.

\subsection{Background information}

We shall now discuss the model of subgraph discovery from graph transactions and coverage patterns.

\subsubsection{Model of subgraph discovery}

In the literature, several efforts $[4,8]$ are being made to discover the knowledge of subgraphs from the given set of graph transactions. In particular, in the area of bioinformatics, research efforts $[22,42]$ have been made by modeling chemical compounds as graph transactions. By considering a given chemical compound as a single unit, the corresponding graph transaction represents chemical elements as vertices and chemical bonds among them as edges. We first present the notion of graph transactions and briefly explain the subgraph discovery approach, which was presented in [42].

A graph transaction $G=(V, E, L, l)$ is a labeled, connected, simple and undirected graph, where $V$ is a set of vertices, $E \subseteq V^{2}$ is a set of edges, $L$ is a set of labels and $l: V \cup E \rightarrow L$, where $l$ is a function for assigning labels to vertices and edges. A graph transactional dataset (GTD) $D$ comprises $n$ such graph transactions, where the value of $n$ is typically large. Notably, a vertex/edge may belong to multiple graph transactions in $D$. A portion $S$ of a graph transaction $G$ is called a subgraph of $G$. Given $G=(V, E)$ and $S=\left(V_{S}, E_{S}\right)$, we say that $S$ is a subgraph of $G$ or $S$ exists in $G$ (denoted as $S \subseteq G)$, iff $V_{s} \subseteq V, E_{s} \subseteq E, \forall(u$, $v) \in E_{s} \longrightarrow u, v \in V_{s}$.

Example 1 Consider a sample chemical compound shown in Fig. 1a and its equivalent graph transaction $G=(V, E, L, l)$ depicted in Fig. 1b. Here, $V=\left\{v_{1}, v_{2}, \ldots, v_{13}\right\}, E=\left\{\left(v_{1}, v_{3}\right)\right.$, $\left.\left(v_{2}, v_{3}\right), \ldots,\left(v_{9}, v_{13}\right)\right\}$ and $L=\{C, F, H, N, O, 1,2,3\}$. A mapping function $l$ maps the vertices $v_{1}, v_{2}, v_{3}, \ldots, v_{13}$ to $H, N, N, \ldots, F$ and the edges $\left(v_{1}, v_{3}\right),\left(v_{2}, v_{3}\right), \ldots,\left(v_{9}, v_{13}\right)$ to $1,3, \ldots, 1$, respectively.

Given a GTD, a subgraph is a potential subgraph if it is present in certain percentage of graphs in GTD. We can 


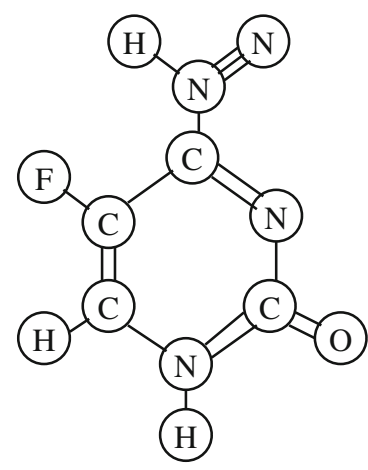

(a)

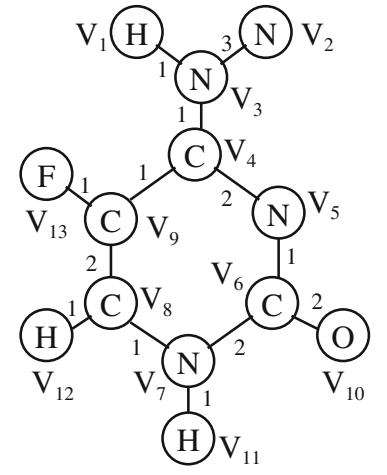

(b)
Fig. 1 a Sample chemical compound, b equivalent graph model

employ a subgraph discovery algorithm (e.g., gSpan [42]) for extracting candidate subgraphs from $D$. The gSpan algorithm employs a depth-first search (DFS) strategy to extract all subgraphs without candidate generation. It uses a patterngrowth approach to build a hierarchical search tree called the DFS Code Tree. In the DFS Code Tree, every node represents a subgraph/graph, and any subgraph/graph in GTD can find its node in the DFS Code Tree. Each node in the tree is assigned with a lexicographical canonical label called the DFS code and one subgraph can have multiple DFS codes. The first DFS code in pre-order traversal over the DFS Code Tree is called the minimum DFS code and is assigned as the canonical label to the subgraph. Moreover, $g$ Span also prunes all the nodes that contain non-minimum DFS code, thereby reducing the size of the DFS Code Tree. A depth-first search over the DFS Code Tree extracts all minimum DFS codes of all candidate subgraphs in $D$. The performance of $g$ Span improves drastically due to the merging of isomorphism test and subgraph growth into one procedure.

\subsubsection{Model of coverage patterns}

We shall now explain the concept of coverage patterns $[18,36]$. Coverage patterns are characterized by the notions of relative frequency, coverage support and overlap ratio. Given a transactional database $D$, each transaction is a subset of a set $I$ of $m$ items $\left\{i_{1}, i_{2}, i_{3}, \ldots, i_{m}\right\} . T^{i_{k}}$ denotes the set of transactions in which item $i_{k}$ is present. The fraction of transactions containing a given item $i_{k}$ is designated as Relative Frequency of $i_{k}\left(R F\left(i_{k}\right)\right)$. Hence, $R F\left(i_{k}\right)=\frac{\left|T^{i} k\right|}{|D|}$. A given item is considered as frequent if its relative frequency is greater than that of a threshold value, which we designate as $\min R F$. A pattern $P$ is a subset of items in $I$, i.e., $P \subseteq I$ where $P=\left\{i_{p}, i_{q}, \ldots, i_{r}\right\}$, where $l \leq p, q, r \leq \mathrm{m}$. The Coverage Set $(\operatorname{CSet}(P))$ of a pattern $P$ is the set of all the transactions that contain at least one item from the pattern $P$, i.e., $\operatorname{CSet}(P)=T^{i_{p}} \cup T^{i_{q}} \cup \ldots \cup T^{i_{r}}$. The Coverage Support of a pattern $P(C S(P))$ is the ratio of the size of $\operatorname{CSet}(P)$ to the size of $D$, i.e., $C S(P)=\frac{|\operatorname{CSet}(P)|}{|D|}$. In order to add a new item to the pattern $P$ such that the coverage support increases significantly, the notion of overlap ratio is introduced. (This is possible in the case when the number of transactions, which are common to the new item and the pattern $P$, is low.) Given a pattern $P$, the notion of overlap ratio of $P$ satisfies the sorted closure property [28], when the items in $P$ are sorted in decreasing order of their relative frequencies, i.e., $l \leq R F\left(i_{p}\right) \leq R F\left(i_{q}\right) \leq \ldots \leq$ $R F\left(i_{r}\right)$. The Overlap Ratio of a pattern $P(O R(P))$ is the ratio of the number of transactions that are common between $C \operatorname{Set}\left(P-i_{r}\right)$ and $T^{i_{r}}$ to the number of transactions in $T^{i_{r}}$, i.e., $O R(P)=\frac{\left|C \operatorname{Set}\left(P-i_{r}\right) \cap\left(T^{i_{r}}\right)\right|}{\left|T^{i^{r}}\right|}$. A high value of coverage support indicates more number of transactions and a low value of overlap ratio means less repetitions among the transactions. A pattern is interesting if its coverage support is greater than or equal to the user-specified minimum Coverage Support threshold value (minCS) and its overlap ratio is less than or equal to the user-specified maximum Overlap Ratio threshold value $(\max O R)$. Given the values of $\min R F, \min C S$ and maxOR, a pattern $P=\left\{i_{p}, i_{q}, \ldots, i_{r}\right\}$ is considered as a coverage pattern if $R F\left(i_{k}\right) \geq \min R F \forall i_{k} \in P, C S(P) \geq \min C S$ and $O R(P) \leq \max O R$. By exploiting the sorted closure property of overlap ratio, a level-wise apriori-based approach has been proposed in [36] and a pattern-growth-based approach has been proposed in [18] for extracting all coverage patterns from $D$, given $\min R F, \min C S$ and $\max O R$ values. Additionally, a MapReduce-based algorithm to extract coverage patterns has been proposed in [32].

To extract the knowledge of coverage patterns, the following heuristics can be followed for setting $\min R F, \min C S$ and maxOR values. Normally, the coverage patterns with maximum coverage $(100 \%)$ and minimum overlap ratio $(0 \%)$ are interesting. Moreover, the coverage patterns having items with less relative frequency are not interesting. So, $\min R F$ threshold value can be set based on the characteristics of the application. In the beginning, as a heuristic, coverage patterns can be extracted by setting $\max O R$ at 0 and $\min C S$ at 1 and $\operatorname{minRF}$ can be set to $50 \%$ of $\min C S$ value. Then, based on the requirement of the number of coverage patterns, maxOR can be increased gradually, while $\min C S$ and $\min R F$ can be decreased gradually.

\section{Proposed framework of the problem}

Consider a graph transactional dataset (GTD) $D$, a minimum relative frequency threshold $\min R F_{g}$, a minimum coverage support threshold $\min C S_{g}$ and a maximum overlap threshold max $O_{g}$. Our proposed SIFT framework returns the set of all subgraph coverage patterns ( $S C P S$ ) subject to the $\min R F_{g}$, $\min C S_{g}$ and $\max _{g}$ constraints. Note that we employ the 
Table 1 Summary of notations

\begin{tabular}{ll}
\hline Notation & Description \\
\hline$G_{i}$ & Graph transaction \\
$D$ & Graph transactions dataset (GTD) \\
$\Psi$ & Universe of subgraphs \\
$S P$ & Subgraph pattern \\
$O_{h}$ & Subgraph identifier (SID) \\
$f_{i}$ & Flat transaction \\
$D_{f}$ & Flat transactional dataset \\
$X$ & Set of SIDs or pattern \\
$S C P S$ & Subgraph coverage patterns \\
\hline
\end{tabular}

notations $\min R F_{g}, \min C S_{g}$ and $\max O_{g}$, (i.e., we add a subscript $g$ to $\min R F$, min $C S$ and $\max O$ ), to represent minimum relative frequency, minimum coverage support and maximum overlap thresholds concerning a graph transactional dataset. Now we shall explain the relevant terminology to present the framework of the problem. Table 1 depicts the summary of notations used in this paper.

\subsection{Subgraph pattern, cover and cover set}

Recall the notions of graph transaction, graph transactional dataset and subgraph from the discussions in Sect. 2.2. Given a GTD $D$ and the set $\Psi$ of all possible subgraphs over $D$, a subgraph pattern $(S P)$ is a set of subgraphs belonging to $\Psi$. Consider a graph transaction $G_{i} \in D$. A subgraph $S_{j}$ is said to cover $G_{i}$ if $S_{j}$ exists in $G_{i}$. We define $\operatorname{cover}\left(S_{j}, G_{i}\right)$ as follows:

$\operatorname{cover}\left(S_{j}, G_{i}\right)=\left\{\begin{array}{l}1 \text { if } S_{j} \subseteq G_{i} \\ 0 \quad \text { otherwise }\end{array}\right.$

Computation of $\operatorname{cover}\left(S_{j}, G_{i}\right)$ involves solving the subgraph isomorphism problem [25], which is NP-complete [16]. The gSpan algorithm [42] uses a canonical labeling system called DFS lexicographical order, which assigns minimum DFS code to each graph as the canonical label. We compute cover $\left(S_{j}, G_{i}\right)$ based on DFS codes. The Cover Set of $S_{j}\left(C \operatorname{Set}_{g}\left(S_{j}, D\right)\right)$ is defined as the set of all graph transactions covered by $S_{j}$. Formally, $\operatorname{CSet}_{g}\left(S_{j}, D\right)=$ $\left\{G_{i} \mid \operatorname{cover}\left(S_{j}, G_{i}\right)=1 \& G_{i} \in D\right\}$. The Cover Set of $S P$ $\left(C \operatorname{Set}_{g}(S P, D)\right)$ is a set of all graph transactions, which are covered by at least one subgraph of $S P$. It is equal to the union of all graph transactions covered by all the subgraphs in $S P$. Hence, $C \operatorname{Set}_{g}(S P, D)=\bigcup_{\forall S_{j} \in S P} C \operatorname{Set}_{g}\left(S_{j}, D\right)$.

\subsection{Relative frequency $R F_{g}$ of a subgraph}

Given $D$ and $S_{j}$, we denote the percentage of graph transactions in $D$ covered by $S_{j}$ as relative frequency $R F_{g}$ of $S_{j}$. We compute $R F_{g}\left(S_{j}, D\right)$ as follows:

$R F_{g}\left(S_{j}, D\right)=\frac{\left|C \operatorname{Set}\left(S_{j}, D\right)\right|}{|D|}$

Here, $0 \leq R F_{g}\left(S_{j}, \mathrm{D}\right) \leq 1$. We can extract subgraphs of interest from $D$ based on user-specified minimum relative frequency $\left(\min R F_{g}\right)$ threshold.

Example 2 Consider a sample graph transactional dataset $D$ comprising of 10 graph transactions $G_{1}$ to $G_{10}$, shown in Fig. 2a. Three subgraphs $S_{1}, S_{2}$ and $S_{3}$ are shown in Fig. 2b. Here, $S_{1}$ is a subgraph of $G_{1}, G_{6}$ and $G_{10} ; S_{2}$ is a subgraph of $G_{5}, G_{7}$ and $G_{8}$; and $S_{3}$ is a subgraph of $G_{4}$ and $G_{7}$. The subgraph $S_{1}$ is said to cover $G_{1}$ since $S_{1} \subseteq G_{1}$. Hence, $\operatorname{cover}\left(S_{1}, G_{1}\right)=1$. Moreover, $C \operatorname{Set}\left(S_{1}, D\right)=\left\{G_{1}, G_{6}, G_{10}\right\}$ and $R F_{g}\left(S_{1}, D\right)=\frac{\left|C \operatorname{Set}\left(S_{1}, D\right)\right|}{D}=\frac{3}{10}=0.3$. Similarly, RF values of $S_{2}$ and $S_{3}$ are 0.3 and 0.2 , respectively.

\subsection{Coverage support}

Given $D$ and a subgraph pattern $S P$, the coverage support of $S P\left(C S_{g}(S P, D)\right)$ is the percentage of graph transactions in $D$ covered by at least one subgraph in $S P$. We compute $C S_{g}(S P, D)$ as follows:

$C S_{g}(S P, D)=\frac{|C \operatorname{Set}(S P, D)|}{|D|}$

Here, $0 \leq C S_{g}(S P, D) \leq 1$. Notably, $C S_{g}(S P, D)=1$ when all of the graph transactions in $D$ are covered by $S P$. Conversely, $C S_{g}(S P, D)=0$ when none of the graph transactions are covered by $S P$. A pattern $S P$ is interesting w.r.t coverage perspective if $C S_{g}(S P, D) \geq \min C S_{g}$, where minC $S_{g}$ is a user-defined minimum coverage support threshold for graph transactions.

\subsection{Overlap}

A pattern $S P$, which satisfies a given $\min C S_{g}$ constraint, may not be interesting if there is significant overlap among the sets of transactions covered by subgraphs of $S P$. In several applications, an $S P$ with maximum coverage support and minimum overlap could be interesting. We now explain the notion of overlap $g_{g}$ for capturing the overlap associated with graph transactions.

In the literature, the concept of overlap between sets is most often described using Euler diagrams [5]. Consider two sets $A$ and $B$ in a universe of objects. The overlap of $A$ and $B$ is computed by $\frac{A \cap B}{A \cup B}$. This equation does not consider the 


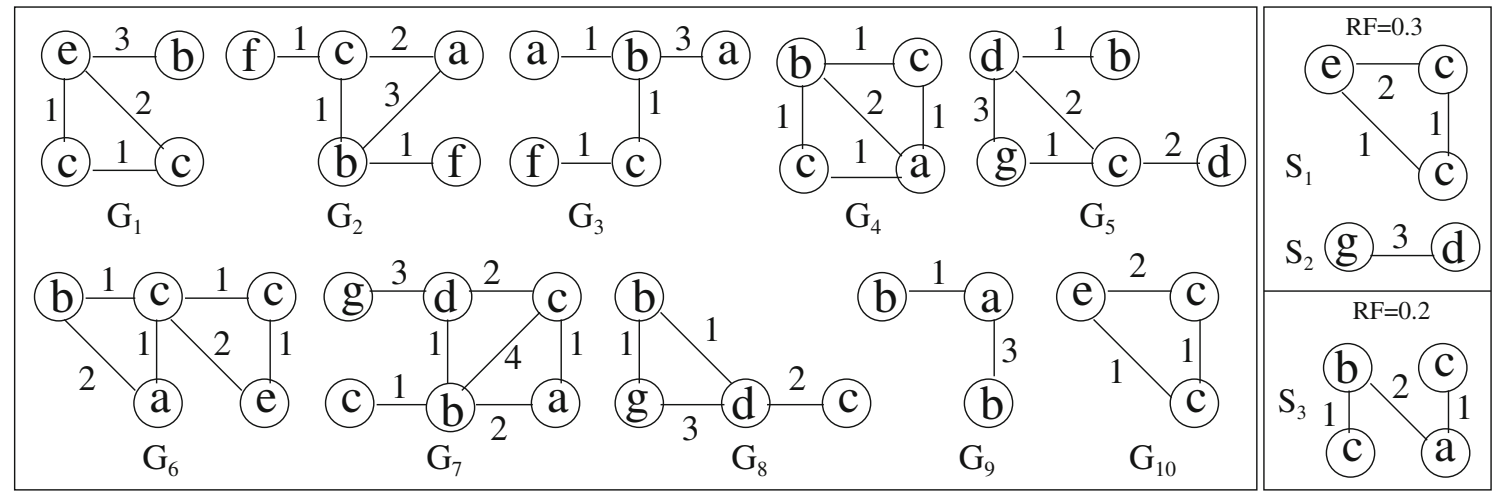

(a)

(b)

Fig. 2 a Sample of 10 graph transactions, $\mathbf{b}$ candidate subgraphs with $\min R F_{g}=0.2$

number of times an object is appearing in either $A$ or $B$. As a result, it is not possible to attach a physical meaning unless we know the nature of repetition of objects in $A$ and $B$. In this paper, we present the notion of overlap by considering the average number of times an object can appear in the given multi-set (contains duplicate elements). Let $M(S P, D)$ be the multi-set, which contains all transactions (with duplicate entries) covered by each subgraph in $S P$. We define the value of overlap $_{g}$ as the average number of times a transaction is repeated in $M(S P, D)$. We define overlap $_{g}$ as follows:

$\operatorname{overlap}_{g}(S P, D)=\left(\frac{|M(S P, D)|}{|C S e t(S P, D)|}-1\right) \cdot 100$

Observe that the value of overlap $g$ can exceed $100 \%$ as the size of $|M(S P, D)|$ could be unbounded. In this paper, we restrict the size of $|M(S P, D)|$ by considering that a transaction can only appear twice in $|M(S P, D)|$. Hence, the notion of overlap $_{g}$ denotes the average number of times a subgraph appears at most twice in $D$. Here, overlap $_{g}=1$ if every transaction appears twice in $M(S P, D)$, i.e., the maximum value of $|M(S P, D)|=2 \cdot|C \operatorname{Set}(S P, D)|$. Conversely, overlap $_{g}=0$ if every transaction appears only once in $M(S P, D)$, i.e., the minimum value of $|M(S P, D)|=|C \operatorname{Set}(S P, D)|$. Hence, $0 \leq \operatorname{overlap}_{g}(S P, D) \leq 1$. A pattern $S P$ is interesting if overlap $(S P, D) \leq \max O_{g}$, where $\max O_{g}$ is a userdefined maximum Overlap threshold for graph transactions.

In essence, there can be different ways of computing overlap based on the application requirement. In case of applications, in which a transaction appears more than twice, say $k$ times, in $|M(S P, D)|$, Equation 4 is modified as $\operatorname{overlap}_{g}(S P, D)=\frac{1}{k-1}\left(\frac{|M(S P, D)|}{|\operatorname{CSet}(S P, D)|}-1\right) \cdot 100$, where the maximum value of $|M(S P, D)|$ equals $k$. $|C \operatorname{Set}(S P, D)|$.

\subsection{Subgraph coverage pattern}

We consider an $S P$ as interesting if the cover set of all subgraphs of $S P$ satisfies the $\min R F_{g}$ threshold, overlap of $S P$ satisfies the $\max O_{g}$ threshold and coverage support of $S P$ satisfies the $\min C S_{g}$ threshold. We designate such $S P s$ as subgraph coverage patterns ( $S C P S$ ). The definition of $S C P$ is given below.

Definition 1 (Subgraph coverage pattern (SCP)) Consider $D$ and a pattern $S P$. We call $S P$ as a subgraph coverage pattern if $C S_{g}(S P, D) \geq \min C S_{g}$ and overlap $g(S P, D) \leq$ $\max O_{g}, \forall S_{j} \in S P, R F_{g}\left(S_{j}, D\right) \geq \min R F_{g}$.

Example 3 In Fig. 2b, let $S P$ be the set $\left\{S_{1}, S_{2}, S_{3}\right\}$. The RF values of $S_{1}, S_{2}$ and $S_{3}$ are $0.3,0.3$, and 0.2 , respectively. The coverage set of $S P, C \operatorname{Set}(S P, D)=\left\{\left(G_{1}, G_{4}, G_{5}, G_{6}, G_{7}, G_{8}\right.\right.$, $\left.G_{10}\right\}$. The coverage support of $S P, C S_{g}(S P, D)=\frac{|C \operatorname{Set}(S P, D)|}{|D|}$ $=\frac{7}{10}=0.7$. The multi-set of transactions covered by pattern $S P, M(S P, D)=\left\{\left(G_{1}, G_{6}, G_{10}\right),\left(G_{5}, G_{7}, G_{8}\right),\left(G_{4}, G_{7}\right)\right\}$. Therefore, the overlap among transactions covered by subgraphs of $S P$, overlap $_{g}(S P, D)=\left(\frac{|M(S P, D)|}{|\operatorname{CSet}(S P, D)|}-1\right)=\left(\frac{8}{7}-\right.$ $1)=0.142$. Given the values of $\min R F_{g}=0.2, \operatorname{minC} S_{g}=0.7$ and $\max O_{g}=0.5$, the pattern $S P=\left\{S_{1}, S_{2}, S_{3}\right\}$ is an $S C P$.

\subsection{Problem statement}

Given a graph transactional dataset $D$, and the values of user-defined constraint parameters $\min R F_{g}, \min C S_{g}$ and max $O_{g}$, the problem is to extract all subgraph coverage patterns satisfying these user-defined constraints.

It can be noted that the objective is to extract SCPS with high coverage value for a given application scenario. Normally, the SCPS having subgraphs with low relative frequency value are not interesting. So, there will be significant number of $S C P S$, which cover small portion of GTD. As min $C S$ threshold increases, the number of $S C P S$ will reduce. Similarly, as minRF increases, the number of SCPS will 
reduce. Regarding overlap, we consider that the $S C P s$ with minimum overlap will be interesting. Therefore, in a dense data set scenario, a smaller number of SCPS will be returned for lower value of overlap. As overlap threshold increases, the number of $S C P S$ explodes.

\section{Proposed SIFT framework}

This section discusses our proposed SIFT framework.

\subsection{Basic idea}

Given a GTD $D$ and the threshold values of $\min R F_{g}$, $\min C S_{g}$ and $\max _{g}$ as input, the goal is to extract all the $S C P s$ from $D$.

A brute-force approach would be to extract all of the possible subgraphs of $D$ based on $\min R F_{g}$, and then determine $C S_{g}$ and overlap $_{g}$ for each combination of subgraphs by computing the corresponding $\mathrm{CSet}_{g}$ values of the given pattern. Each combination of subgraphs of $D$ could be a candidate $S C P$. The number of candidate $S C P$ s formed by the subgraphs of even a small number of graph transactions would essentially explode, thereby making the extraction of $S C P$ s extremely challenging and difficult to scale.

The basic idea is as follows. We convert the given graph transactions into the corresponding flat transactions. For this purpose, we extract all subgraphs from GTD and assign unique Subgraph IDentifiers (SID) to each subgraph. Next, we convert each graph transaction into flat transaction by including the corresponding SID. Next, we propose an efficient methodology to extract $S C P S$ from flat transactional dataset. We shall henceforth refer to this framework as subgraph ID-based flat transactional (SIFT) framework.

We can intuitively understand that SIFT provides opportunities for efficient determination of candidate sets. Further, it provides efficient way to compute coverage and overlap for each candidate set. This is because by considering each graph transaction as a set of SIDs, the coverage and overlap of a given subgraph pattern can be calculated through a set-based operation. Thus, we are essentially replacing complex and computationally expensive graph-based operations by set-based operations, which are typically faster by several orders of magnitude. Hence, the problem of extracting SCPS becomes the problem of extracting combinations of SIDs from the set of flat transactions. Thus, we propose a pattern mining-based extraction method by exploiting an overlaprelated pruning heuristic, which we shall discuss now.

Incidentally, $C S_{g}$ and overlap $_{g}$ threshold constraints do not satisfy the downward closure property [21]. However, we can exploit the overlap ratio measure proposed in [36] for extracting coverage patterns from a flat transactional dataset. The overlap ratio constraint satisfies sorted closure prop-

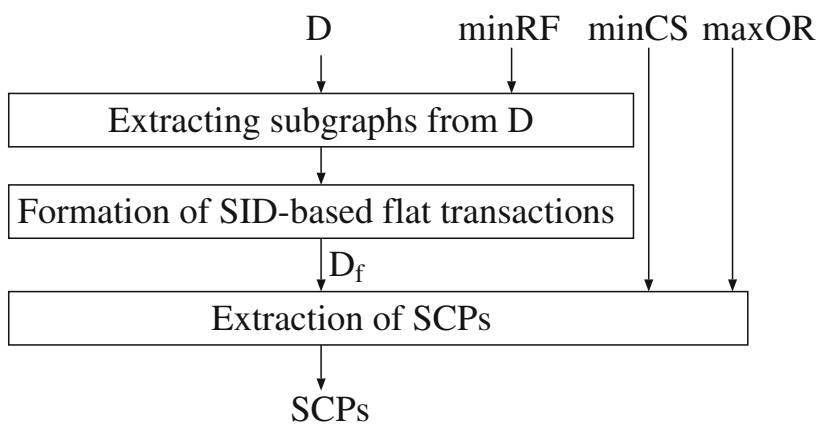

Fig. 3 Details of the SIFT framework

erty [28]. Consider a candidate pattern $S P=\left\{S_{p}, S_{q}, \ldots, S_{r}\right\}$, where the subgraphs in $S P$ are sorted in descending order of their relative frequencies. When overlap ratio of $S P$ fails to satisfy the maximum overlap ratio threshold, any superset of $S P$ cannot possibly satisfy the maximum overlap threshold. Hence, we can avoid generating supersets of $S P$. We use this heuristic in our proposed approach for effective pruning of candidate patterns. The steps to extract $S C P$ s from flat transaction are as follows. First, we sort all of the candidate subgraphs in descending order of their relative frequencies. Then, starting from individual candidate subgraph as $S P$, we continue to generate candidate $S P$ of progressively larger sizes, while using the pruning heuristic based on sorted closure property of overlap ratio to efficiently prune candidate $S P$.

\subsection{Details of the SIFT framework}

Given $D, \min R F_{g}, \min C S_{g}$ and $\max O_{g}$, our proposed $S I F T$ framework extracts $S C P$ s from $D$. Figure 3 depicts the details of the SIFT framework. SIFT framework consists of the following steps (Algorithm 1):

(i) Extracting subgraphs from $D$

(ii) Formation of SID-based flat transactions and

(iii) Extraction of $S C P s$

We shall now explain these steps.

\subsubsection{Extracting subgraphs from $D$}

Based on the $\min R F_{g}$ threshold, a subgraph discovery algorithm $g$ Span [42] is used to extract all the subgraphs from $D$ subject to $\min R F_{g}$ constraint (refer Sect. 2.2 for gSpan algorithm). We construct the set $S G$ of subgraphs using gSpan algorithm, where each subgraph $S_{j}$ is of the form $<$ Clabel, CSet>, where Clabel represents canonical label of $S_{j}$ and CSet consists of all GIDs of graph transactions that contains subgraph $S_{j}$. 


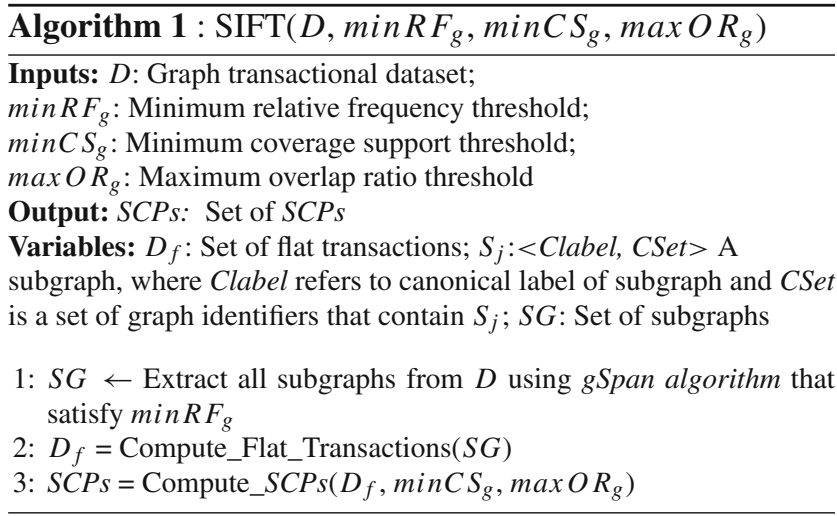

\subsubsection{Formation of SID-based flat transactions}

The input to this step is a set of subgraphs $S G$ of the form $<$ Clabel, CSet $>$. In this step, we form the flat transaction for each graph transaction in $D$. The flat transaction contains the SIDs corresponding to GID. The details are given in Algorithm 2. In Algorithm 2, we maintain two hashmaps SubList: $<$ SID, Clabel $>$ and $D_{f}:<f_{i}, S(S I D)>$, where $f_{i}$ represents $i^{\text {th }}$ flat transaction identifier and $S(S I D)$ represents set of SIDs corresponding to the $i^{\text {th }}$ graph transaction. For every subgraph $S_{j}$ in $S G$, we check if the canonical label of $S_{j}$ exists in SubList.Clabel. If it does not exist, we assign a new SID to $S_{j}$ and insert SID, Clabel into SubList. Otherwise, we assign the SID to $S_{j}$ corresponding to Clabel of $S_{j}$ in SubList.Clabel (see Lines 2-9). In both the cases, for each subgraph $S_{j}$ and for each GID in CSet of $S_{j}$, we insert SID of $S_{j}$ into set $S(S I D)$ of flat transaction identifier $f_{i}$ corresponding to GID (see Lines 9-10). The set $<f_{i}, S(S I D)>$ forms the $S I D$-based flat transactional dataset $D_{f}$ (see Line 14)).

The mapping of subgraphs in graph transaction $G_{i}$ to SIDs in flat transaction $f_{i}$ is a bijective function $\mathcal{F}$ represented as follows:

$\forall S_{j} \in G_{i}, \forall O_{h} \in f_{i}, \mathcal{F}: S_{j} \rightarrow O_{h}$

where $O_{h}$ is an SID of $S_{j}$. When $G_{i}$ has no subgraphs, $f_{i}=\{\phi\}$. Note that there are no duplicate SIDs in any flat transaction. The elements in each flat transaction are nothing but SID of subgraphs extracted from $D$ subjective to the $\min R F_{g}$ constraint. The constructed flat transactions do not represent all the features of original graph transaction, but represent only the subgraphs which satisfy $\min R F_{g}$ constraint.

Let $\Psi_{S I D}=\left\{O_{1}, O_{2}, \ldots, O_{m}\right\}$ be the set of $m$ distinct SIDs in $D_{f}$. Let $D_{f}=\left\{f_{1}, f_{2}, f_{3}, \ldots, f_{n}\right\}, \forall f_{i} \in D_{f}, f_{i} \subseteq \Psi_{S I D}$, the set $D_{f}$ forms the flat transactional dataset, where $f_{i}$ is corresponding flat transaction of $G_{i}, \forall i=1$ to $n$.

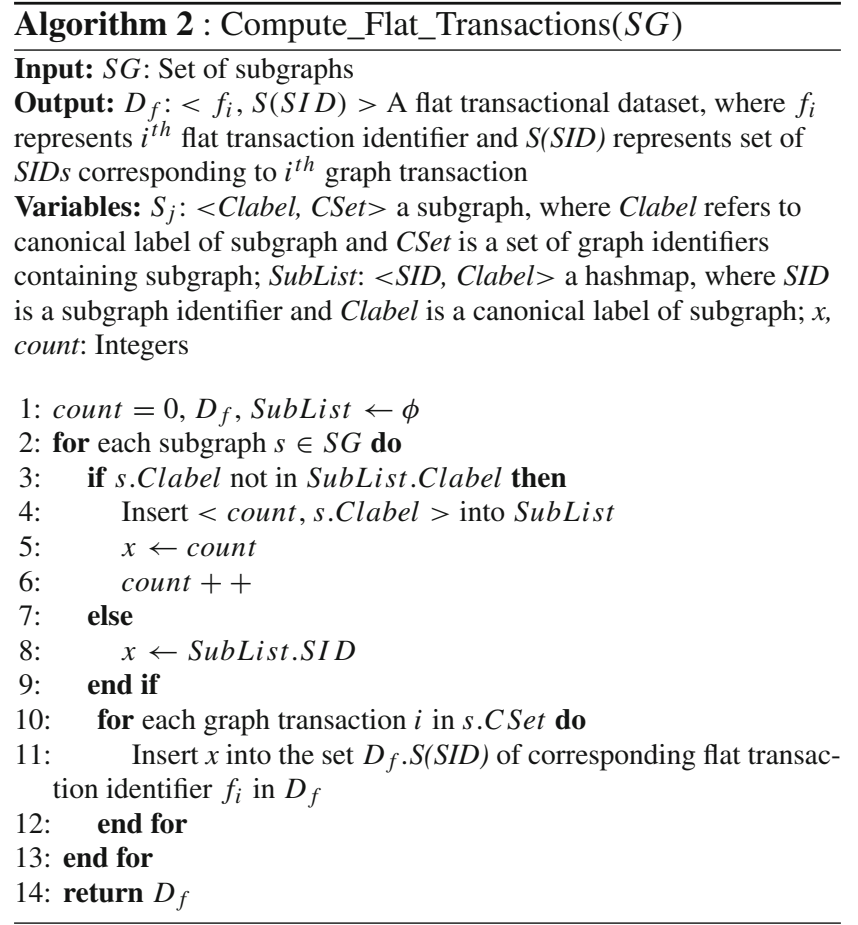

\subsubsection{Extraction of SCPs}

After converting GTD into SID-based flat transactional dataset, our objective is to extract $S C P S$ subject to the constraints of $\min R F_{g}, \min C S_{g}$ and $\max O_{g}$. In this section, we explain the process to extract $S C P S$ subject to the $\min C S_{g}$ and $\max O_{g}$ constraints.

Under a brute-force approach, we would need to compute the values of $C S_{g}(S P, D)$ and overlap $_{g}$ for a prohibitively large number of candidate patterns formed by all SIDs. This is because the $\min C S_{g}$ and $\max O_{g}$ constraints do not satisfy the downward closure property [21]. However, we can exploit the overlap ratio measure proposed in [18] for extracting coverage patterns from a flat transactional dataset. The overlap ratio measure satisfies the sorted closure property [28]. As explained in Sect. 2.2, the coverage pattern mining algorithm extracts coverage patterns subject to the constraints of minimum relative frequency $(\min R F)$, minimum coverage support $(\min C S)$ and maximum overlap ratio $(\max O R)$.

Now, we explain the equivalence between the $\min R F$, $\min C S$ and $\max O R$ constraints for flat transactions (presented in Sect. 2.2 as defined in [18]) and $\min R F_{g}, \min C S_{g}$ and max $O_{g}$ constraints associated with $S C P S$ (defined in Sect. 3).

Recall that for flat transactions, the notion of relative frequency $R F\left(i_{k}\right)$ of an item $i_{k}$ is the percentage of transactions, which contain $i_{k}$. In case of GTD, $R F_{g}\left(S_{j}, D\right)$ denotes the percentage of graph transactions, which contain a subgraph $S_{j}$. Furthermore, for flat transactions, the notion of coverage support $C S(X)$ of a pattern $X$ is the percentage of the union of transactions covered by each item of $X$. In case of 
GTD, $C S_{g}(S P, D)$ of a subgraph pattern $S P$ denotes the percentage of the union of graph transactions covered by each subgraph of $S P$.

Regarding the overlap aspect, we have defined overlap $_{g}$ concept and $\max O_{g}$ constraint for GTD. First, we explain the overlap ratio $(O R)$ constraint, which has been defined to extract coverage patterns for flat transactions [36]. Next, we explain how to employ the $O R$ constraint to extract $S C P S$ subject to the $\max O_{g}$ constraint.

Given a pattern $X$, and if the elements in $X$ are sorted in the descending order of their relative frequency values, Overlap Ratio $(O R(X))$ of a pattern $X$, which satisfy the sorted closure property. We shall explain the sorted closure property after defining the overlap ratio of the pattern. The notion of CSet of a pattern has been explained in Sect. 2.2.

Definition 2 (Overlap ratio of a pattern $X$ ) Let $X=$ $\left\{O_{p}, O_{q}, \ldots, O_{r}, O_{s}\right\}$ be a pattern such that $R F\left(O_{p}\right) \geq$ $R F\left(O_{q}\right) \geq \cdots \geq R F\left(O_{r}\right) \geq R F\left(O_{s}\right)$. (Here, the notations $O_{p}, O_{q}, O_{r}$ and $O_{s}$ represent SIDs.) The overlap ratio of a pattern $X$ is defined as the ratio of the number of transactions common in $C \operatorname{Set}\left(X-\left\{O_{s}\right\}\right)$ and $C \operatorname{Set}\left(O_{s}\right)$ to $C \operatorname{Set}\left(O_{s}\right)$. It is defined as follows:

$$
O R(X)=\frac{\left|C \operatorname{Set}\left(X-\left\{O_{s}\right\}\right) \cap C \operatorname{Set}\left(O_{s}\right)\right|}{\left|\operatorname{CSet}\left(O_{s}\right)\right|}
$$

For a pattern $X, 0 \leq O R(X) \leq 1$. A pattern $X$ is interesting if $O R(X) \leq \max O R$, where $\max O R$ is a user-defined maximum Overlap Ratio threshold. A pattern $X$ is said to be non-overlap pattern if $O R(X) \leq \max O R$ and $R F\left(O_{h}\right) \geq$ $\min R F, \forall O_{h} \in X$. Incidentally, it can be observed that the $\max O R$ constraint follows the sorted closure property, which is explained below.

Definition 3 (Sorted closure property) Let the pattern $X=\left\{O_{p}\right.$, $\left.O_{q}, \ldots, O_{r}, O_{s}\right\}, 1 \leq p \leq q \leq r \leq s \leq m$ such that the items in $X$ are sorted in the descending order of their relative frequency values, i.e., $R F\left(O_{p}\right) \geq R F\left(O_{q}\right) \geq \cdots \geq$ $R F\left(O_{r}\right) \geq R F\left(O_{s}\right)$. If $O R(X)$ is less than or equal to $\max O R$, i.e., $O R(X) \leq \max O R$, all the non-empty subsets of $X$ containing $O_{s}$ will also have $O R$ less than or equal to $\max O R$.

Suppose, we extract the set $S$ of coverage patterns from a given GTD with $O R(X) \leq \alpha$. We can compute overlap $_{g}(X)$ for all $X \in S$ and extract coverage patterns with $\operatorname{overlap}_{g}(X) \leq \alpha$. For a given pattern $X$, the relationship between $O R$ and overlap $_{g}$ is given in Theorem 1.

Theorem 1 Consider a coverage pattern $X=\left\{O_{1}, O_{2}, \ldots\right.$, $\left.O_{p}\right\}$ with $O R(X) \leq \alpha$. Then, overlap $g(X) \leq \alpha$, when $p \leq\left(\frac{1+\alpha}{\alpha}\right)$.
Proof From the definition of overlap $_{g}$ in Sect. 3,

$\operatorname{overlap}_{g}(X)=\left(\frac{|M(X)|}{|C \operatorname{Set}(X)|}-1\right)$

As $X$ is a coverage pattern, $R F\left(O_{1}\right) \geq R F\left(O_{2}\right) \geq \cdots \geq$ $R F\left(O_{p}\right)$. We consider a worst case scenario and assume that $\left|C \operatorname{Set}\left(O_{1}\right)\right|=\left|C \operatorname{Set}\left(O_{2}\right)\right|=\ldots=\mid C \operatorname{Set}\left(O_{p} \mid=t\right.$. So, $M(X)=p . t$ and $C \operatorname{Set}(X)=p . t-(p-1) \alpha t$. Substituting the values of $M(X)$ and $C \operatorname{Set}(X)$ in Equation 5 ,

$$
\left(\frac{p . t}{p . t-(p-1) \alpha t}-1\right)=\frac{\alpha(p-1)}{p-\alpha(p-1)}
$$

By equating the above equation to $\alpha$ and solving for $p$,

$\frac{\alpha(p-1)}{p-\alpha(p-1)}=\alpha ; \Longrightarrow p=\frac{1+\alpha}{\alpha}$

Thus, we conclude that for a pattern $X$, when $O R(X) \leq \alpha$, $\operatorname{overlap}_{g}(X) \leq \alpha$ if $p \leq\left(\frac{1+\alpha}{\alpha}\right)$.

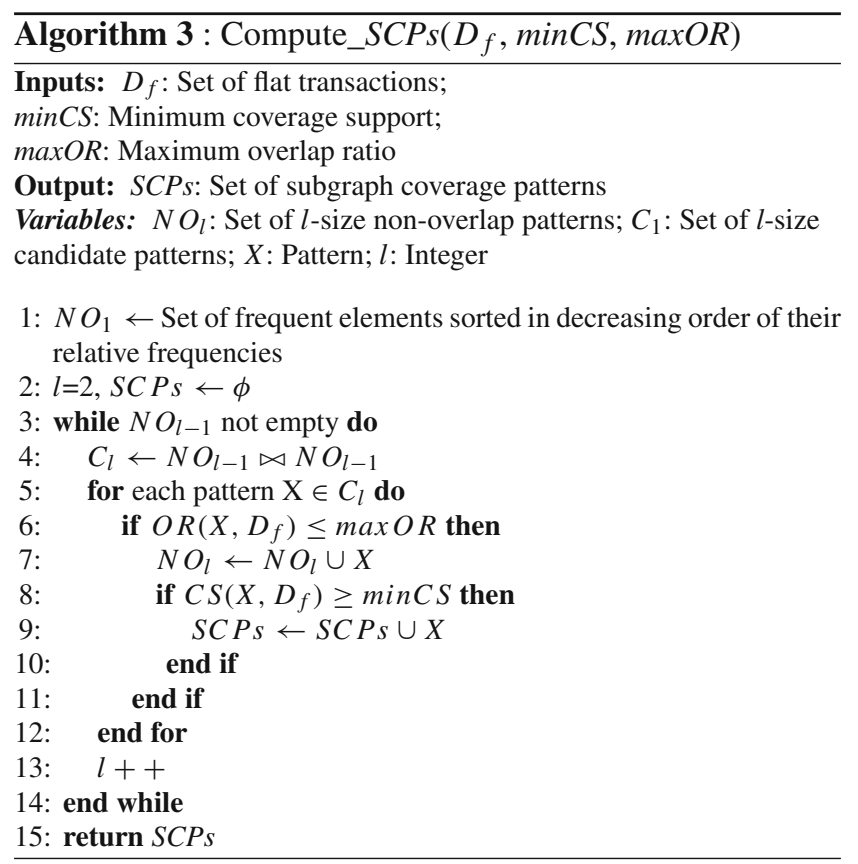

Notably, we have employed two notions (overlap $g$ and overlap ratio) to capture the notion of overlap. The notion of overlap $_{g}$ is intuitive from the user perspective, whereas overlap ratio (and maxOR) was employed as a pruning measure for efficient extraction of SCPs. For extracting SCPs, we can employ an existing coverage pattern algorithm such as a level-wise pruning based approach $[32,36]$ or a patterngrowth approach [18], with the value of $\min C S$ equal to $\min C S_{g}$ and the value of $\max O R$ equal to $\max O_{g}$. 
For extracting SCPs from flat transactional dataset, we employ coverage pattern mining algorithm proposed in [36]. Algorithm 3 depicts the coverage pattern mining algorithm. The inputs are flat transactional dataset $D_{f}$ and user parameters $\min C S$ and $\max O R$ values. Coverage pattern mining algorithm exploits apriori like level-wise search approach to find the $l$-size candidate patterns from $(l-1)$-size non-overlap patterns (see Lines 3-4). A non-overlap pattern is a pattern that satisfies maxOR constraint. It uses sorted closure property to prune the search space and extracts all non-overlap patterns, which become the candidates for the next iteration (see Lines 5-7). The considered non-overlap patterns that satisfy the minCS constraint are considered as the SCPS (see Lines 8-9). This process is repeated until no new non-overlap patterns are generated.

After extracting the set of $S C P s$, top- $k S C P s$ can be listed by considering a ranking criteria based on $C S_{g}$ or a combination of $C S_{g}$ and overlap $g$ values of $S C P s$ based on the specific requirements of the application domain.

\subsection{Time complexity}

The time complexity of the proposed SIFT framework is equals:

$O(k m n+r m)+O(m q)+\sum_{l=1}^{m} l\left(\left|C_{l-1}\right| \cdot\left|C_{l-1}\right|\right)$

where $O(\mathrm{kmn}+\mathrm{rm})$ is the complexity of subgraph extraction, $O(\mathrm{mn})$ is the time complexity of flat transactions modeling, and $\sum_{l=1}^{m} l\left(\left|C_{l-1}\right| \cdot\left|C_{l-1}\right|\right)$ is the time complexity of SCPS computation. The explanation of each term in Equation 7 is as follows:

First, in SIFT framework, we employ gSpan algorithm to extract subgraphs from GTD. As mentioned in [2], the complexity of gSpan algorithm to extract all subgraphs from GTD is $O(k m n+r m)$, where $k$ is the maximum number of subgraph isomorphism tests, $m$ is the number of frequent subgraphs, $n$ is the number of graph transactions, and $r$ is the maximum number of duplicate codes of the frequent subgraphs that grow from other minimum DFS codes. It can be noted that extraction of all subgraphs from a given graph transaction is an NP-complete problem [2]. To improve performance, the gSpan algorithm employs the notion of minimum DFS code and converting the subgraph extraction problem into a pattern mining problem through string comparison. In practical scenarios, the value of $m \leq n$, the value of $r$ is much less than $n$ and the value of $k$ is small for sparse and diverse labels. Hence, the time complexity for subgraph extraction depends on the value of $m \times n$.

Second, the process to compute the flat transactional dataset from the set of <canonical label of a subgraph, set of the corresponding GIDs > produced in the preceding step consists of two steps. First, we assign an SID to each unique canonical label (Clabel) and compute an hashmap $<$ SID, Clabel $>$. The search time for the existence of Clabel in the hashmap take $O(1)$. Second, after mapping the Clabel with unique $S I D$, for each corresponding $G I D$, we will insert $S I D$ into the corresponding flat transaction identifier. The search time to insert is $O(1)$. Consider that on average, each SID belongs to $q$ number of transactions. The time complexity to compute the flat transactional dataset is bounded by $O(m \times q)$. Notably, $q \ll m$. Therefore, the time complexity to model flat transactions from graph transactions is proportional to $m$.

Third, in SIFT framework, we employ an iterative levelwise apriori based algorithm. The time complexity of an iterative pruning algorithm is $\sum_{l=1}^{m} l\left(\left|C_{l-1}\right| \cdot\left|C_{l-1}\right|\right)$, where $\left|C_{l-1}\right|$ is the number of candidate patterns of size $l$ (Refer to Chapter 6 of [38]). In the proposed SIFT framework, the number of candidate patterns generated depends on the value of overlap ratio threshold maxOR. Normally, at lower values of maxOR, less number of candidate patterns are produced at each level.

Overall, the time complexity of proposed SIFT framework depends on the graph transactional dataset size $n$, number of subgraphs extracted $m$ and number of candidate patterns generated. Note that the value of $m$ depends on $\min R F$ threshold and the number of candidate patterns depends on maxOR threshold. By choosing proper values of $\min R F$ and $\max O R$ threshold values, it is indeed capable of extracting subgraph coverage patterns from graph transactional dataset.

\section{Performance evaluation}

We conducted our experiments in the ADA cluster [1] (at IIIT Hyderabad), which consists of 42 Boston SYS-7048GR-TR nodes equipped with dual Intel Xeon E5-2640 v4 processors, providing 40 virtual cores per node. The aggregate theoretical peak performance of ADA is 47.62 TFLOPS. We have conducted experiments on 20 virtual machines. Each virtual machine is allocated with 2 GB memory. We also reported the experiments on the scalability aspect of our proposed approach by varying the number of virtual machines from 5 to 40. We implemented our proposed schemes in Python 3.0. The link to the code for the implementation is provided in the footnote. ${ }^{1}$

We used three real datasets, namely Yeast 167 (Yeast anticancer), P388 (Leukemia), from Pubchem $[3,43]$ and Zinc dataset consisting of drug-like molecules [37]. The Yeast 167 and P388 datasets consist of chemical compounds, which are modeled as graph transactions. In these datasets, each

\footnotetext{
${ }^{1}$ https://github.com/srinivas2234/SCPs.
} 
Table 2 Summary of the real datasets

Table 3 Parameters of the SIFT framework performance evaluation

\begin{tabular}{llllll}
\hline Dataset & \#graph transactions & Avg. density & \#vertex labels & \#edge labels & Avg. size of graph \\
\hline Yeast & 79601 & 0.0537 & 75 & 3 & 40.7 \\
P388 & 41472 & 0.052 & 73 & 3 & 41.8 \\
Zinc & 4672 & 0.73 & 15 & 10 & 1.8 \\
\hline
\end{tabular}

\begin{tabular}{llllll}
\hline Parameter & Default & & & Variations \\
\cline { 2 - 3 } \cline { 5 - 6 } & P388, Yeast & Zinc & & P388, Yeast & Zinc \\
\hline minRF & 0.3 & 0.025 & & $0.3-1$ (step size $=0.1)$ & $0.025-1$ (step size $=0.05,0.1)$ \\
maxOR & 0.3 & 0.5 & & $0-1($ step size $=0.1)$ & $0-1($ step size $=0.1)$ \\
minCS & 0.7 & 0.7 & & $0.3-1($ step size $=0.1)$ & $0-1($ step size $=0.1)$ \\
$N_{M}$ & 20 & 20 & & $5-40($ step size $=5)$ & NIL \\
\hline
\end{tabular}

chemical compound is modeled as graph, where chemical elements are represented as vertices and chemical bonds among them are represented as edges. We have reported our case study by considering the Zinc dataset. The Zinc dataset consists of drug-like molecules docked with $1 \mathrm{WOF}$ protein to form a protein-ligand complex. Table 2 summarizes the three datasets.

To the best of our knowledge, there exists no other approach for extracting SCPs from a GTD. As the number of subgraphs increases, the complexity of a naïve brute-force approach for extracting SCPS increases exponentially as it requires the determination of the coverage and overlap values based on prohibitively expensive graph-based computations. Hence, in the absence of any meaningful reference approach for comparison, we define the objective of our performance evaluation toward demonstrating the feasibility of the proposed SIFT framework in extracting SCPS from a given dataset.

We have conducted the experiments by implementing three components of the SIFT framework as follows. First, we employ the gSpan algorithm [42] for extracting all candidate subgraphs from a given GTD and assign SIDs to the extracted subgraphs. Second, we employ the proposed SIFT framework to form the transformed flat transactional dataset over the extracted SIDs. Third, to extract SCPs from the transformed flat transactions, we use the MapReduce-based coverage pattern mining algorithm [32], which was proposed to extract coverage patterns from flat transactions. Table 3 summarizes the parameters of our performance study.

The performance metrics for extracting SCPS are (i) processing time $\left(T_{S}\right)$ to extract subgraphs, assign SIDs and form SID-based flat transactions, (ii) number of candidate subgraphs $\left(N_{S}\right)$, (iii) average number of SIDs $(A V G)$ in the $S I D$-based flat transactions, (iv) processing time $\left(T_{S C P}\right)$ to extract SCPS from flat transactions, (v) number of patterns $\left(N_{P}\right)$ to be examined for extracting SCPs and (vi) number of $S C P S\left(N_{S C P}\right)$. Here, $T_{S}$ represents the processing time con- sumed to extract subgraphs by accessing the graph dataset from the disk. $T_{S C P}$ is the processing time consumed for extracting SCPS from the SID-based transactional dataset, which resides on disk.

\subsection{Effect of varying $\min R F$}

The results in Fig. 4 depict the effect of varying $\min R F$. The results in Fig. 4a indicate the performance of $T_{S}$, while the results in Fig. 4b show the performance of $N_{S}$ as we vary $\min R F$ for the P388 and Yeast datasets. The results in Fig. 4a show that when the value of $\operatorname{minRF}$ is low, the value of $T_{S}$ is high. As $\min R F$ is increased, the value of $T_{S}$ reduces exponentially due to the pruning effect of $\min R F$. The value of $T_{S}$ depends upon the number of subgraphs extracted from the dataset. The results in Fig. $4 \mathrm{~b}$ show that the number of SIDs decreases with increase in the value of $\min R F$. This occurs due to decrease in the number of subgraphs that satisfy the $\min R F$ constraint. The results in Fig. 4c depict the effect of varying minRF on $A V G$. The results show that when the value of $\min R F$ is low, the value of $A V G$ is high, and as $\min R F$ increases, the value of $A V G$ is decreased. This is because at lower value of $\min R F$, there will be a large number of subgraphs, which satisfy the $\min R F$ constraint. As the value of $\min R F$ increases, the number of subgraphs decreases because less number of subgraphs satisfy the $\min R F$ constraint. The value of $A V G$ depends on the number of subgraphs that are extracted. Therefore, at lower values of $\min R F$, a transactional dataset with large $A V G$ is extracted.

The results in Figs. 4d, e and $\mathrm{f}$ show that the values of $T_{S C P}, N_{P}$ and $N_{S C P}$ decrease with the increase in the value of $\min R F$, respectively. The reason is that at lower value of $\min R F$, there will be large number of subgraphs satisfying the $\min R F$ constraint. As minRF increases, the value of $N_{P}$ decreases because less number of patterns satisfy the $\min R F$ constraint. Consequently, the values of $T_{S C P}$ and $N_{S C P}$ also decrease. 


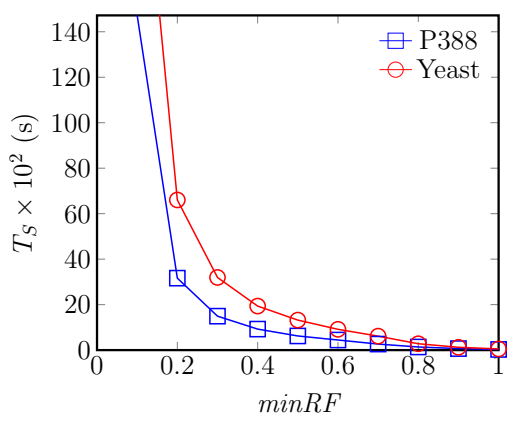

(a) $T_{S}$

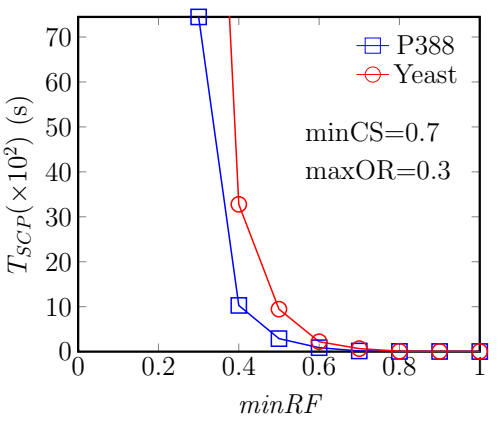

(d) $T_{S C P}$

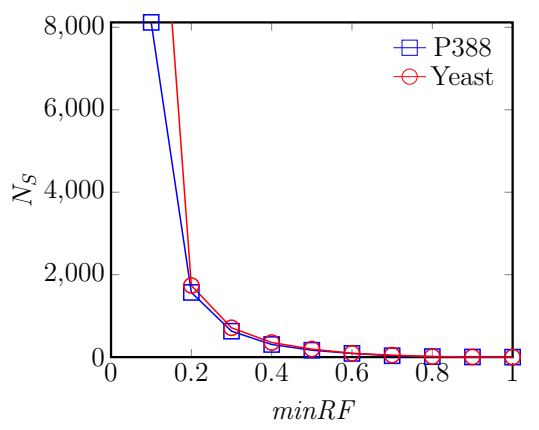

(b) $N_{S}$

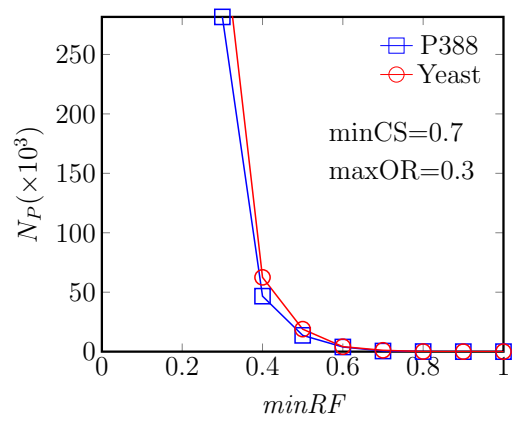

(e) $N_{P}$

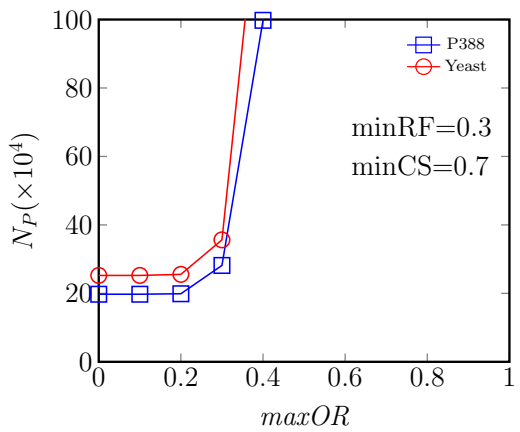

(b) $N_{P}$

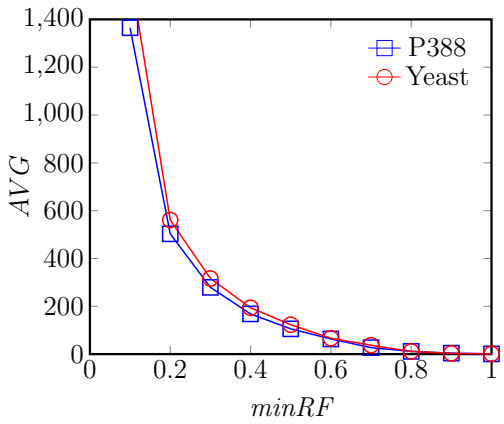

(c) $A V G$

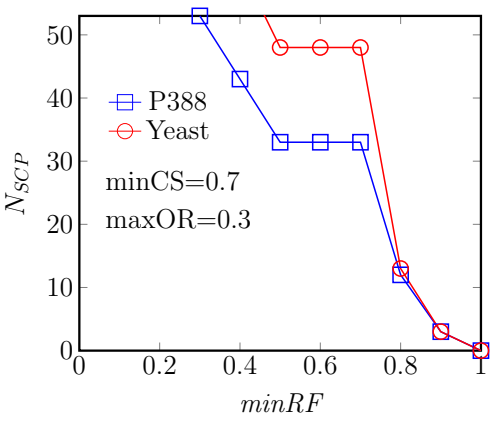

(f) $N_{S C P}$

Fig. 4 Effect of varying $\operatorname{minRF}$

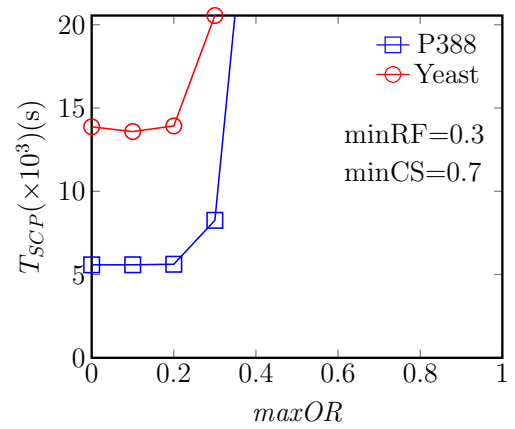

(a) $T_{S C P}$

Fig. 5 Effect of varying $\max O R$

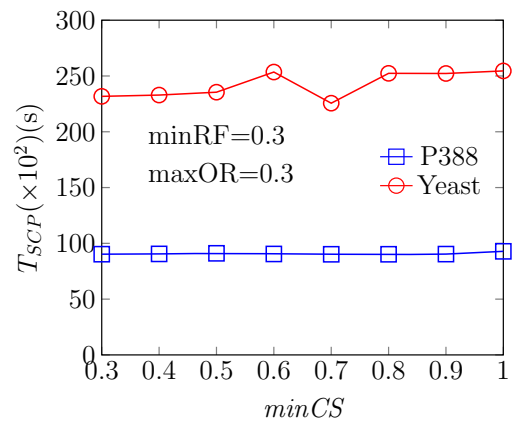

(a) $T_{S C P}$

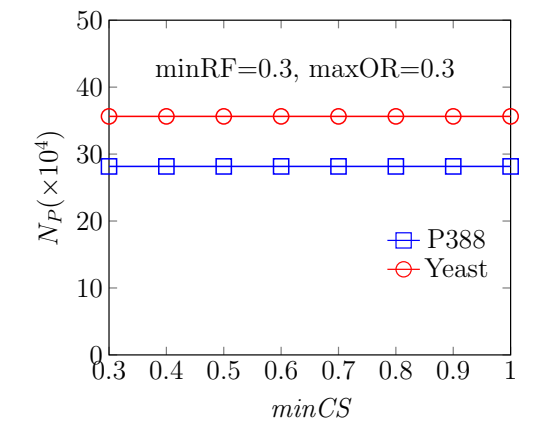

(b) $N_{P}$

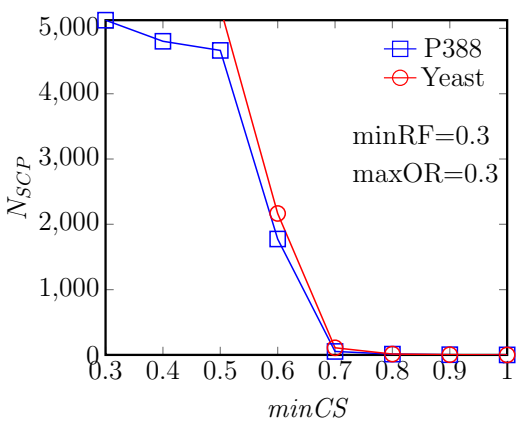

(c) $N_{S C P}$

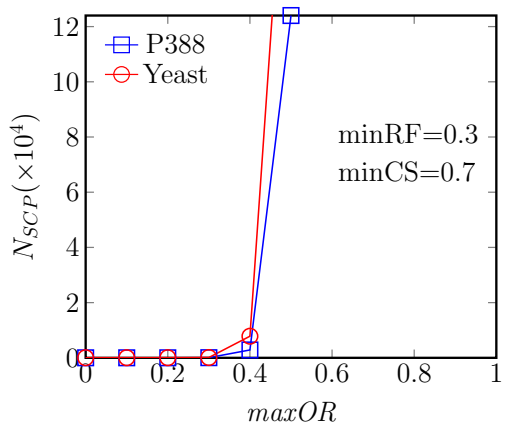

(c) $N_{S C P}$

Fig. 6 Effect of varying $\min C S$ 


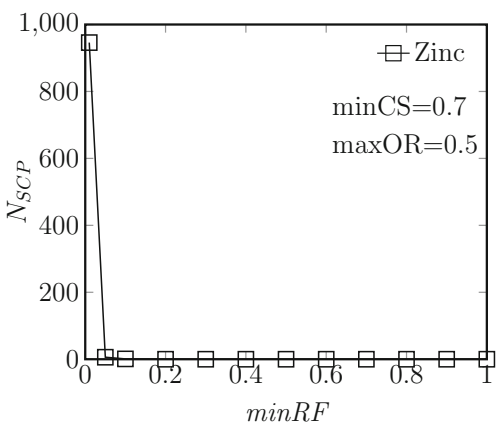

(a) $\min R F$ vs $N_{S C P}$

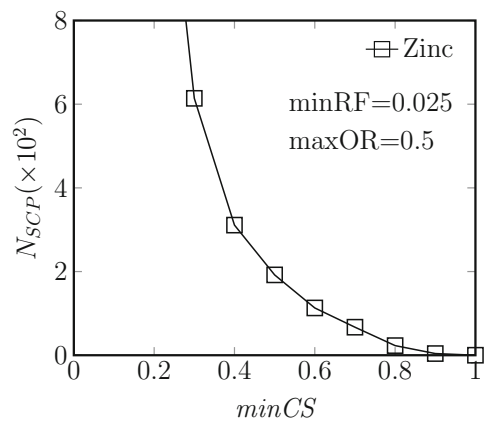

(b) $\min C S$ vs $N_{S C P}$

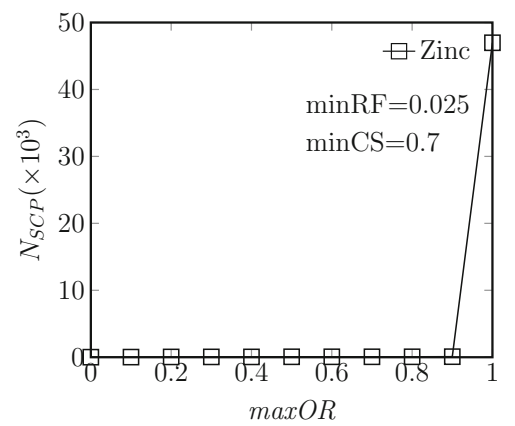

(c) $\max O R$ vs $N_{S C P}$

Fig. 7 Effect of varying $\operatorname{minRF}, \min C S$ and $\max O R$ on Zinc dataset

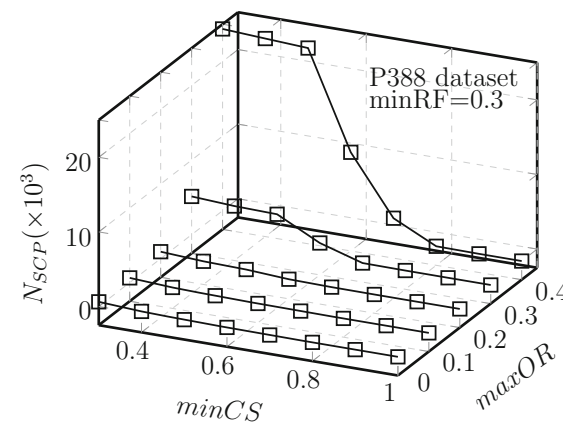

(a) $\min C S$ vs $\max O R$ vs $N_{S C P}$

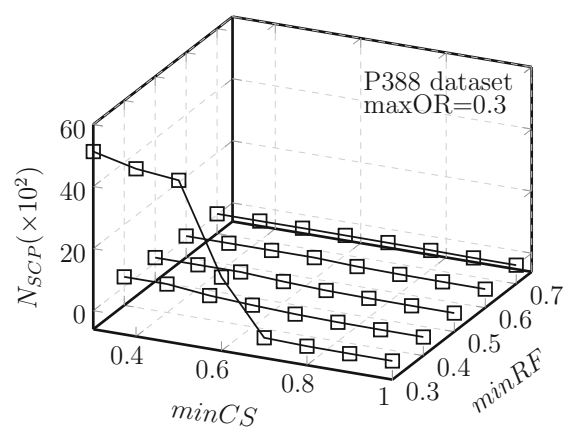

(b) $\min C S$ vs $\operatorname{minRF}$ vs $N_{S C P}$

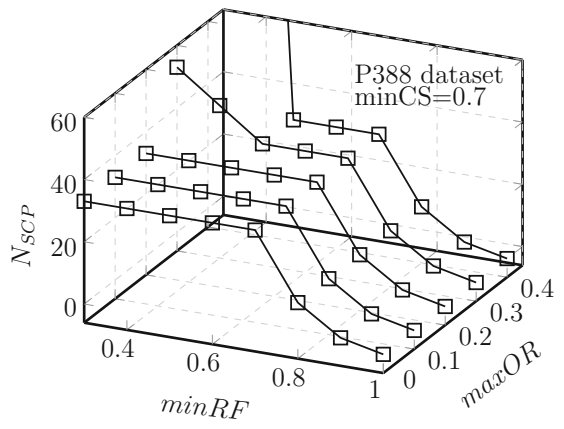

(c) $\operatorname{minRF}$ vs $\max O R$ vs $N_{S C P}$

Fig. 8 Effect of varying (a) $\min C S$ and $\max O R \mathbf{b} \min C S$ and $\min R F$ and $\mathbf{c} \min R F$ and $\max O R$

It can be observed that we have reported results starting from $\min R F=0.1$ as we could not experiment with $\min R F$ less than 0.1 due to explosion in the number of patterns.

\subsection{Effect of varying maxOR}

The results in Fig. 5 depict the effect of varying the value of $\max O R$. The results in Figs. 5a, b and c show that $T_{S C P}, N_{P}$ and $N_{S C P}$ increase with the increase in $\max O R$, respectively. The reason is that at lower value of $\operatorname{maxOR}$, there will be less number of patterns, which satisfy the $\max O R$ constraint, thereby resulting in less value of $T_{S C P}$ and $N_{S C P}$. As maxOR increases, the value of $N_{P}$ increases because more patterns satisfy the maxOR constraint, which increases the value of $T_{S C P}$ and $N_{S C P}$. It can be observed that even at $\max O R=0$, there are 48 SCPs in Yeast and 13 SCPs in P388. At higher values of maxOR, more number of $S C P S$ can be extracted.

\subsection{Effect of varying $\operatorname{minCS}$}

The results in Fig. 6 depict the effect of varying the value of $\min C S$. The results in Fig. $6 \mathrm{a}$ and $\mathrm{b}$ indicate that the values of $T_{S C P}$ and $N_{P}$ remain comparable for all the values of $\min C S$ for both datasets. The reason is as follows. When the values of minRF and maxOR are fixed, the same number of candidate patterns is examined to extract the SCPs. Therefore, as expected, irrespective of variations in the value of $\min C S$, both the values of $T_{S C P}$ and $N_{P}$ remain comparable.

The results in Fig. 6c indicate that the value of $N_{S C P}$ decreases with increase in the value of $\min C S$. Notably, in the proposed approach, after satisfying the maxOR constraint, we prune a candidate pattern if it does not satisfy the $\min C S$ constraint. At higher values of $\min C S$, a candidate pattern will be pruned even though it satisfies the maxOR constraint. As a result, the value of $N_{S C P}$ reduces as we increase the value of $\min C S$.

The results in Figs. $7 \mathrm{a}-\mathrm{c}$ depict the effect of varying the values of $\min R F$, $\min C S$ and $\max O R$, respectively, for Zinc dataset. The results depict trends similar to P388 and Yeast datasets. The value of $N_{S C P}$ is small due to the small size of Zinc dataset.

\subsection{Performance results with 3D plots}

The results in Fig. 8a depict the effect of varying the values of $\min C S$ and $\operatorname{maxOR}$. The result shows that when the values of $\min C S$ and $\max O R$ are low, the value of $N_{S C P}$ is small due to candidate pruning based on $\max O R$ constraint. 
When the values of $\min C S$ are high and $\max O R$ is low, the value of $N_{S C P}$ decreases further because very few patterns satisfy high value of $\min C S$ and low value of $\max O R$. When the value of $\min C S$ is low and $\operatorname{maxOR}$ is high, the value of $N_{S C P}$ is high because more number of patterns satisfy low value of $\min C S$ and high value of $\operatorname{maxOR}$. However, when the values of $\min C S$ and $\max O R$ are high, the value of $N_{S C P}$ will decrease because there are few patterns that may satisfy the high value of $\min C S$.

The results in Fig. 8b depict the effect of varying the values of $\min C S$ and $\min R F$. The result shows that at low values of $\min C S$ and $\min R F$, the value of $N_{S C P}$ is high. This is because, large number of candidate patterns will be generated at lower values of $\min R F$ and most of them satisfy the low value of $\min C S$ constraint. When $\min C S$ is high and $\min R F$ is low, the value of $N_{S C P}$ low because, less number of candidate patterns satisfy the high value of $\min C S$ threshold. At low values of $\min C S$ and high values of $\min R F$, the value of $N_{S C P}$ is low, due to small number of candidate pattern generation. Further, when the values of $\min R F$ and $\min C S$ are high, the value of $N_{S C P}$ decreases further.

The results in Fig. 8c depict the effect of varying the values of $\max O R$ and $\operatorname{minRF}$. The results show that $N_{S C P}$ does not vary much at lower values of $\min R F$ and $\max O R$. When we increase the value of $\min R F$, the value of $N_{S C P}$ decreases due to decrease in number of candidate pattern. When the values of $\min R F$ and $\max O R$ are high, the value of $N_{S C P}$ is less, due to less number of candidate patterns. However, when the values of minRF and maxOR are high, the number of $S C P S$ explodes due to large number of candidate pattern generation.

\subsection{Effect of varying $N_{M}$}

Figure 9 depicts the effect of varying the number $N_{M}$ of machines. Observe that the value of $T_{S C P}$ decreases with increase in the value of $N_{M}$. This is due to increase in the parallel extraction of SCPS. However, the change in the value of $T_{S C P}$ decreases with increase in $N_{M}$ and exhibits a saturation effect when more than 30 machines are used. This is due to communication overhead. The results show that the value of $T_{S C P}$ can be reduced by employing additional resources.

Given a dataset, the processing time to extract $S C P$ s equals the sum of the processing time to form SID-based graph transactions (as depicted in Figs. 4a) and the processing time to extract SCPS (as depicted in Fig. 9). Overall, the results demonstrate that it is feasible to extract the knowledge of $S C P S$ by processing a reasonable size dataset of Yeast with 79601 graph transactions.

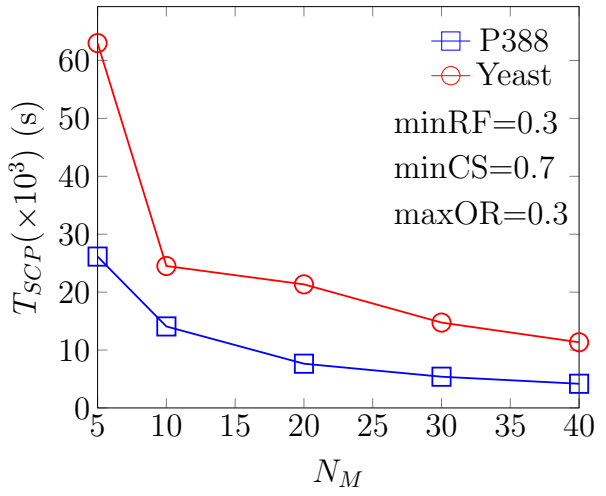

Fig. 9 Effect of varying $\mathbf{N}_{M}$

\subsection{Discussion about setting thresholds in SIFT}

In this approach, conversion of graph transactions into SIDbased flat transactions is one time computation process. Once graph transactions are transformed to flat transactions, it is always possible to choose relative frequency thresholds greater than $\min R F_{g}$ and compute $S C P s$ for various values of $\min C S_{g}$ and $\max O R_{g}$.

Now let us discuss how to set the values of the parameters such as $\min R F_{g}, \min C S_{g}$ and $\max O R_{g}$. The $\min R F_{g}$ threshold value can be set to half the maximum $\min R F_{g}$ value. Then, based on number of coverage patterns, $\min R F_{g}$ can be decreased. The goal is to extract $S C P S$ with maximum coverage, while minimizing the overlap to zero. Hence, as a heuristic, we could start with the coverage support value equal to 1 and then progressively keep decreasing the value of coverage support until a desired number of $S C P S$ is obtained. Regarding $\max O R_{g}$, we can start with $\max O R_{g}$ equal to 0 and then progressively keep increasing the value of $\max O R_{g}$ until a desired number of SCPS can be obtained.

Based on the application, the domain expert can first extract $S C P$ s by setting $\max O R=0$ and $\min C S=1$. If the domain expert needs more number of $S C P s$, he can increase $\max O R$ or decrease minCS progressively. Normally, the process of pattern mining is an iterative approach. As we have proposed a pattern mining model, the usual methodologies employed to set threshold values can be employed in this case also.

\section{Case study: usefulness of SCPs in drug design}

We demonstrate the feasibility of applying knowledge of $S C P S$ in computer-aided drug design toward developing a drug for coronavirus. Corona viruses that include the SARS coronavirus 2 responsible for the COVID-19 pandemic are pathogens that cause various diseases that are 
Fig. 10 a Sample graph modeling of protein-ligand complex, b candidate subgraphs and corresponding $\operatorname{minRF}$ values

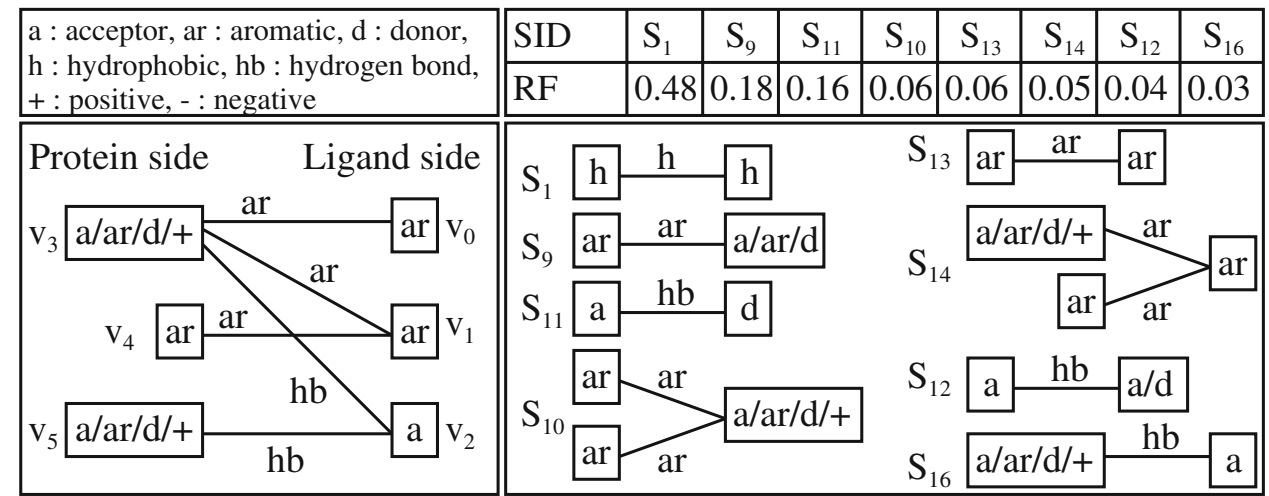

(a) (b)
Table 4 Top 8 SCPs extracted from PLC dataset

\begin{tabular}{llll}
\hline S.No & Subgraph coverage pattern & Coverage support & Overlap ratio \\
\hline 1 & $\left\{S_{1}, S_{9}, S_{11}, S_{12}, S_{16}\right\}$ & 0.9 & 0.25 \\
2 & $\left\{S_{1}, S_{9}, S_{11}, S_{12}\right\}$ & 0.87 & 0.12 \\
3 & $\left\{S_{1}, S_{9}, S_{11}\right\}$ & 0.83 & 0 \\
4 & $\left\{S_{1}, S_{11}, S_{10}, S_{13}, S_{12}, S_{16}\right\}$ & 0.83 & 0.17 \\
5 & $\left\{S_{1}, S_{11}, S_{10}, S_{14}, S_{12}, S_{16}\right\}$ & 0.81 & 0.17 \\
6 & $\left\{S_{1}, S_{11}, S_{13}\right\}$ & 0.72 & 0.0 \\
7 & $\left\{S_{1}, S_{11}, S_{10}\right\}$ & 0.71 & 0.0 \\
8 & $\left\{S_{1}, S_{11}, S_{14}\right\}$ & 0.7 & 0.0 \\
\hline
\end{tabular}

sometimes fatal in human beings. Coronavirus main protease enzymes (CoV-Mpro) are crucial for virus replication. Drugs designed to inhibit this class of enzymes help in treating coronavirus infection [45]. We consider Zinc database comprising 250000 drug-like molecules. We selected Mpro protein (PDB ID: 1WOF) using the Autodock 4.2 software program [30]. Molecular docking procedure takes each of the 250000 molecules, identifies the best binding mode with the protein, and gives the binding affinity and the protein-ligand bound complex (PLC) structure using a scoring function. Better the intermolecular interactions between the protein and the ligand, better is the binding affinity and better is the molecule for it to be a drug. The top-1000 molecules among the 250000 molecules in the initial dataset that yielded high binding affinity with the protein molecule were chosen for mining SCPs.

For our case study, protein-ligand complexes (PLCs) are converted to graphs transactions using GReMLIN [34]. A ligand can interact with the same protein at different sites, producing multiple graphs for the same protein and ligand, but different vertex and edge label sets. Here, vertices are amino acids of proteins and atoms of ligands and the edges are interactions between amino acids and ligands. Example 4 presents the modeling of PLC as a graph transaction.

Example 4 Consider a sample PLC modeled as a graph transactions $G=(V, E, L, l)$ shown in Fig. 10a. The left side part nodes belong to protein and the right side part nodes belong to ligand. Here, $V=\left\{v_{0}, v_{1}, \ldots, v_{5}\right\}, E=\left\{\left(v_{0}, v_{3}\right)\right.$, $\left.\left(v_{1}, v_{3}\right), \ldots,\left(v_{2}, v_{5}\right)\right\}, \quad L=\{$ aromatic, acceptor, acceptor/ aromatic/donor/positive, aromatic bond and hydrogen bond $\}$. A mapping function $l$ maps the vertices $v_{0}, v_{1}, \ldots, v_{5}$ to aromatic, aromatic, ..., acceptor/aromatic/donor/positive and edges $\left(v_{0}, v_{3}\right),\left(v_{1}, v_{3}\right), \ldots,\left(v_{3}, v_{5}\right)$ to aromatic bond, aromatic bond, ..., hydrogen bond $\}$, respectively.

The top-1000 ligands interact with $1 \mathrm{WOF}$ protein and form 1000 protein-ligand complexes. GReMLIN generated 4672 graph transactions from these 1000 PLCs. We extracted $S C P s$ by providing $\min R F=0.025, \min C S=0.7$ and $\max O R=0.5$. The time consumed to extract subgraph coverage patterns is about 10 seconds (3.52 seconds to model flat transaction from graph transactions and 6.03 seconds to extract subgraph coverage patterns from flat transactions). The top- 8 candidate subgraphs along with their corresponding $R F$ values are depicted in Fig. 10b, and the top-8 SCPs sorted by coverage support and their corresponding overlap ratio are provided in Table 4.

Consider an $\operatorname{SCP}\left\{S_{1}, S_{9}, S_{11}\right\}$ that covers $83 \%$ of Zinc dataset with 0 overlap. Figure 11 a depicts the overall structure of the Mpro protein and highlights the region, where a drug molecule could bind. We have analyzed the interactions among all residues that have interactions with at least one of the 1000 ligands in the dataset. The analysis regarding the utility of SCPS in understanding protein-ligand interactions and its possible inputs to drug design efforts is as follows. 

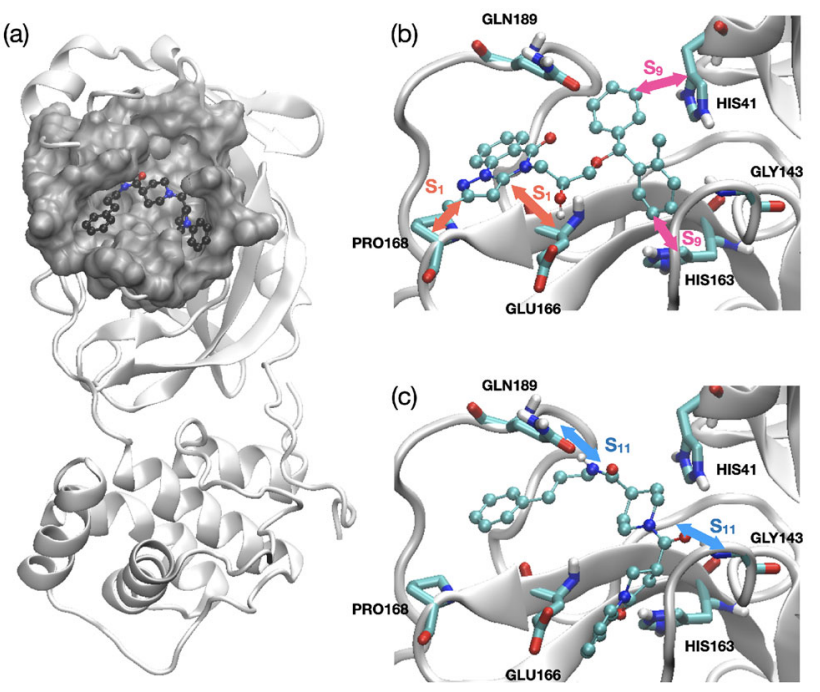

Fig. 11 a Structure of the MPro protein bound to a ligand. b Six selected protein amino acids and interactions with a ligand molecule corresponding to $S_{1}$ and $S_{9}$ subgraphs c Protein-ligand interactions corresponding to $S_{11}$ subgraph

Figure $11 \mathrm{~b}$ depicts an example of a ligand in which interactions corresponding to $S_{1}$ and $S_{9}$ subgraphs are possible (orange and pink arrows). As shown in Fig. 11b, it is evident that the method captured the hydrophobic interaction $S_{1}$ and aromatic interaction $S_{9}$, which contribute toward the favorable binding affinity between the protein and the ligand. On the other hand, Fig. 11c depicts the protein-ligand hydrogen bonding interactions involving another ligand that represent the $S_{11}$ subgraph. Similar to the aromatic and hydrophobic interactions above, the approach captures the hydrogen bonded interaction efficiently. These three subgraphs have an overall coverage of $83 \%$ with overlap ratio as zero indicating their prevalence and hence importance for the molecules to bind to the protein. Such a new knowledge gives possible directions for improving the molecule by modifying the structure of these ligands so that multiple modes of interactions are possible and hence, improve the binding affinities. Therefore, the proposed SIFT framework not only helps in understanding the protein-ligand interactions, but also helps in designing better drugs by extracting the knowledge of subgraph coverage patterns.

\section{Conclusion}

Subgraph pattern mining is an active research area with applications in the domains of chemical, biological and social networks. Given graph transactional data, existing works have focused on the problem of extracting frequent subgraphs, but they have not considered the problem of extracting the knowledge of coverage-related subgraph patterns. Hence, we have introduced the concept of subgraph coverage patterns. In particular, we have proposed the SIFT framework for extracting subgraph coverage patterns from graph transactional data based on $\min R F, \min C S$ and $\max O$ constraints. Our performance evaluation with three real datasets demonstrates the effectiveness of the proposed scheme in terms of processing time and pruning efficiency. We have also demonstrated the feasibility of applying the knowledge of SCPs through a case study in the bioinformatics domain. To the best of our knowledge, this is the first work to consider the extraction of subgraph coverage patterns from graph transactional data. Given the prevalence of graph data modeling, the proposed model of SCPS has a potentially huge scope for opening up new avenues for the extraction of interesting knowledge from graph datasets in several important and diverse domains.

Funding The research of A Srinivas Reddy and P Krishna Reddy is supported by India-Japan Joint Research Laboratory Project entitled "Data Science based farming support system for sustainable crop production under climatic change (DSFS)," funded by Department of Science and Technology, India (DST) and Japan Science and Technology Agency (JST). The research of U Devapriya Kumar is supported by IHub-Data, IIIT Hyderabad.

Data Availability The datasets Yeast 167 (Yeast anti-cancer), P388 (Leukemia) are available at https://sites.cs.ucsb.edu/ xyan/dataset.htm

\section{Declarations}

Conflicts of interest On behalf of all authors, the corresponding author states that there is no conflict of interest.

Code availability The source code is available at https://github.com/ srinivas2234/SCPs.

Ethics approval Not applicable.

Consent to participate Not applicable.

Consent for publication Not applicable.

\section{References}

1. ADA. http://hpc.iiit.ac.in/wiki/index.php/Ada_User_Guide (Accessed in September 2021)

2. UIUC technical report, UIUCDCS-R-2002-2296. https://sites.cs. ucsb.edu/ xyan/papers/gSpan.pdf (Accessed in September 2021)

3. Pubchem. https://pubchem.ncbi.nlm.nih.gov/ (2021)

4. Aida, M., Pieter, M., Wout, B., Pieter, M., Boris, C., Bart Goethals, K.L.: Grasping frequent subgraph mining for bioinformatics applications. BioData Min. 11(1), 1-20 (2018)

5. Alsallakh, B., Aigner, W., Miksch, S., Hauser, H.: Radial sets: interactive visual analysis of large overlapping sets. IEEE Trans. Visual Comput. Gr. 19(12), 2496-2505 (2013)

6. Amiri, A., Salari, M.: Time-constrained maximal covering routing problem. OR Spectrum 41(2), 415-468 (2019)

7. Andrew, G.D., Paola, V.L.: The minimal hitting set generation problem: Algorithms and computation. SIAM 31(1), 63-100 (2017) 
8. Ayed, R., Hacid, M.S., Haque, R., Jemai, A.: An updated dashboard of complete search FSM implementations in centralized graph transaction databases. J. Intell. Inf. Syst. 55, 149-182 (2020)

9. Borgelt, C., Berthold, M.R.: Mining molecular fragments: finding relevant substructures of molecules. In: Proceedings of the ICDM, pp. 51-58 (2002)

10. Charu C, A., Haixun, W.: Managing and mining graph data, vol. 40. Springer (2010)

11. Chen, J., Lin, Y., Li, J., Lin, G., Ma, Z., Tan, A.: A rough set method for the minimum vertex cover problem of graphs. Appl. Soft Comput. 42, 360-367 (2016)

12. Chvatal, V.: A greedy heuristic for the set-covering problem. Math. Oper. Res. 4(3), 233-235 (1979)

13. Cormode, G., Karloff, H., Wirth, A.: Set cover algorithms for very large datasets. In: Proceedings of the ACM CIKM, pp. 479-488 (2010)

14. Dehaspe, L., Toivonen, H., King, R.D.: Finding frequent substructures in chemical compounds. In: Proceedings of the KDD, pp. 30-36 (1998)

15. Fazekas, K., Bacchus, F., Biere, A.: Implicit hitting set algorithms for maximum satisfiability modulo theories. In: Proceedings of the IJCAR, pp. 134-151 (2018)

16. Fortin, S.: The graph isomorphism problem: Technical report. Univ Alberta, Edmonton (1996)

17. Fournier Viger, P., Cheng, C., Lin, J.C.W., Yun, U., Kiran, R.U.: TKG: Efficient mining of top- $k$ frequent subgraphs. In: Proceedings of the Big Data Analytics, pp. 209-226 (2019)

18. Gowtham Srinivas, P., Krishna Reddy, P., Trinath, A.V., Bhargav, S., Uday Kiran, R.: Mining coverage patterns from transactional databases. J. Intell. Inf. Syst. 45, 423-439 (2015)

19. Gramm, J., Guo, J., Hüffner, F., Niedermeier, R.: Data reduction and exact algorithms for clique cover. ACM J. Exp. Algorithm. pp. 2.2-2.15 (2009)

20. Guevara, V.I.G., Calderon, S.G., Cabrera, E.A., Calvo, H.: Symbolic learning for improving the performance of transversalcomputation algorithms. IEEE Access 7, 19752-19761 (2019)

21. Han, J., Cheng, H., Xin, D., Yan, X.: Frequent pattern mining: current status and future directions. Data Min. Knowl. Disc. 15(1), 55-86 (2007)

22. Inokuchi, A., Washio, T., Motoda, H.: An apriori-based algorithm for mining frequent substructures from graph data. In: Proceedings of the European Conference on Principles of Data Mining and Knowledge Discovery, pp. 13-23 (2000)

23. Jiang, C., Coenen, F., Zito, M.: A survey of frequent subgraph mining algorithms. Knowl. Eng. Rev. 28(1), 75-105 (2013)

24. Jiang, H., Wang, H., Philip, S.Y., Zhou, S.: GString: A novel approach for efficient search in graph databases. In: Proceedings of the ICDE, pp. 566-575 (2007)

25. Kuramochi, M., Karypis, G.: Frequent subgraph discovery. In: Proceedings of the ICDM, pp. 313-320 (2001)

26. Li, R., Wang, W.: REAFUM: Representative approximate frequent subgraph mining. In: Proceedings of the ICDM, pp. 757-765. SIAM (2015)

27. Li, Y., Fan, J., Wang, Y., Tan, K.L.: Influence maximization on social graphs: A survey. IEEE TKDE 30(10), 1852-1872 (2018)

28. Liu, B., Hsu, W., Ma, Y.: Mining association rules with multiple minimum supports. In: Proceedings of the ACM SIGKDD, pp. 337-341 (1999)

29. Medina, S.G., Fassio, A.V., de A. Silveira, S., da Silveira, C.H., de Melo-Minardi, R.C.: CALI: A novel visual model for frequent pattern mining in protein-ligand graphs. In: International Conference on Bioinformatics and Bioengineering, pp. 352-358 (2017)

30. Morris, G.M., Huey, R., Lindstrom, W., Sanner, M.F., Belew, R.K., Goodsell, D.S., Olson, A.J.: AutoDock4 and AutoDockTools4: automated docking with selective receptor flexibility. J. Comput. Chem. 30(16), 2785-2791 (2009)
31. Orenstein, Y., Pellow, D., Marçais, G., Shamir, R., Kingsford, C.: Designing small universal $k$-mer hitting sets for improved analysis of high-throughput sequencing. PLoS Comput. Biol. 13(10), 1-15 (2017)

32. Ralla, A., Siddiqie, S., Reddy, P.K., Mondal, A.: Coverage pattern mining based on MapReduce. In: Proceedings of the ACM IKDD CoDS-COMAD, pp. 209-213 (2020)

33. Rehman, S.U., Khan, A.U., Fong, S.: Graph mining: A survey of graph mining techniques. In: Proceedings of the International Conference on Digital Information Management, pp. 88-92 (2012)

34. Ribeiro, V.S., Santana, C.A., Fassio, A.V., Cerqueira, F.R., da Silveira, C.H., Romanelli, J.P.R., Patarroyo-Vargas, A., Oliveira, M.G.A., Gonçalves-Almeida, V., Izidoro, S.C., de Melo-Minardi, R.C., Silveira, S.d.A.: visGReMLIN: Graph mining-based detection and visualization of conserved motifs at $3 \mathrm{D}$ protein-ligand interface at the atomic level. BMC Bioinformatics 21(2), 1-12 (2020)

35. Santana, C.A., Cerqueira, F.R., Da Silveira, C.H., Fassio, A.V., De Melo-Minardi, R.C., Silveira, S.d.A.: GReMLIN: A graph mining strategy to infer protein-ligand interaction patterns. In: IEEE International Conference on Bioinformatics and Bioengineering, pp. 28-35 (2016)

36. Srinivas, P.G., Reddy, P.K., Bhargav, S., Kiran, R.U., Kumar, D.S.: Discovering coverage patterns for banner advertisement placement. In: Proceedings of the PAKDD, pp. 133-144 (2012)

37. Sterling, T., Irwin, J.J.: ZINC 15 - Ligand discovery for everyone. J. Chem. Inf. Model. 55(11), 2324-2337 (2015)

38. Tan, P.N., Steinbach, M., Karpatne, A., Kumar, V.: Introduction to Data Mining, 2nd edn. Pearson (2018)

39. Wagner, M., Friedrich, T., Lindauer, M.: Improving local search in a minimum vertex cover solver for classes of networks. In: Proceedings of the IEEE Congress on Evolutionary Computation, pp. 1704-1711 (2017)

40. Wang, C., Xie, M., Bhowmick, S.S., Choi, B., Xiao, X., Zhou, S.: FERRARI: an efficient framework for visual exploratory subgraph search in graph databases. VLDB J. pp. 1-26 (2020)

41. Wu, J., Li, C.M., Jiang, L., Zhou, J., Yin, M.: Local search for diversified top- $\mathrm{k}$ clique search problem. Computers \& Operations Research 116, 104867 (2020)

42. Xifeng Y., Jiawei H.: gSpan: Graph-based substructure pattern mining. In: Proceedings of the ICDM, pp. 721-724 (2002)

43. Yan, X., Cheng, H., Han, J., Yu, P.S.: Mining significant graph patterns by leap search. In: Proceedings of the ACM SIGMOD, pp. 433-444 (2008)

44. Yan, X., Yu, P.S., Han, J.: Graph indexing: a frequent structurebased approach. In: Proceedings of the ACM SIGMOD, pp. 335$346(2004)$

45. Yang, H., Xie, W., Xue, X., Yang, K., Ma, J., Liang, W., Zhao, Q., Zhou, Z., Pei, D., Ziebuhr, J., Hilgenfeld, R., Yuen, K.Y., Wong, L., Gao, G., Chen, S., Chen, Z., Ma, D., Bartlam, M., Rao, Z.: Design of wide-spectrum inhibitors targeting coronavirus main proteases. PLoS Biol. 3(10), 1742-1752 (2005)

46. Zareie, A., Sheikhahmadi, A., Khamforoosh, K.: Influence maximization in social networks based on TOPSIS. Expert Syst. Appl. 108, 96-107 (2018)

47. Zhefeng, W., Enhong, C., Qi, L., Yu, Y., Yong, G., Biao, C.: Information coverage maximization in social networks. Comput. Res. Repository arxiv:1510.03822 (2015)

48. Zhou, D., Zhang, S., Yildirim, M.Y., Alcorn, S., Tong, H., Davulcu, H., He, J.: A local algorithm for structure-preserving graph cut. In: Proceedings of the ACM SIGKDD, pp. 655-664 (2017)

Publisher's Note Springer Nature remains neutral with regard to jurisdictional claims in published maps and institutional affiliations. 